\title{
Europa, Islam und Koran: Zu einigen Elementen in der gegenwärtigen gesellschaftlichen Debatte
}

\author{
Michael Marx
}

\section{Einleitung}

In der Diskussion zur kulturellen Identität Deutschlands oder Europas wird der Begriff ,jüdisch-christliches Europa“ gelegentlich ins Feld geführt. Der Begriff ist erst nach dem Zweiten Weltkrieg aufgekommen und scheint manchmal dazu zu dienen, den Islam von Europa abzugrenzen. Sicherlich war Religion für die Entstehung der europäischen Kultur von großer Bedeutung, wobei das Christentum stärkeren Einfluss als die jüdische Tradition hatte. Zwar wurde auf der einen Seite die Hebräische Bibel als Altes Testament zu einem Bestandteil der christlichen Tradition, die jüdisch-rabbinische Tradition, die das heutige Judentum prägt, hat auf der anderen Seite für die Prägung Europas wahrscheinlich eine eher kleine Rolle gespielt.

Neben den Bezügen zu religiösen Traditionen sollte nicht übersehen werden, dass es die griechische Antike ist, die seit der Renaissance als prägend für die europäische Zivilisation gesehen wurde. Die Hervorhebung der vorchristlichen griechischen Antike wurde dabei bewusst als Gegengewicht zur Macht des Christentums in Europa gesetzt. Interessanterweise ist die griechische Antike allerdings nicht nur für das Abendland prägend gewesen, sondern hat auch in der islamischen Welt weitergelebt. Die Antike ist dabei fast eine überdehnte Epoche, in unserem Fall würden wir sie im vorchristlichen Jahrtausend bei Homer beginnen lassen und über die Vorsokratiker, Sokrates, Platon und Aristoteles, über die Epoche des Hellenismus hinweg (Alexander der Große und die Ausbreitung der griechischen Kultur im Orient) bis hin zur Übergangsepoche der „Spätantike“ verlaufen lassen.

Mit dem Begriff „Spätantike“ wird für den Mittelmeerraum die Zeit zwischen Antike und Frühmittelalter (ca. 3. bis 7. Jahrhundert) bezeichnet. Diese Übergangszeit wird inzwischen nicht mehr als bloße Zerfallsepoche des römischen 
Reichs, sondern als Entstehungszeit religiöser Traditionen gesehen. In ihr haben sich die christliche Tradition (Kirchenväter, Schriftenkanon, Konzilien etc.) und das rabbinische Judentum (Entstehung des Talmuds) herausgebildet. Doch auch die Entstehung des Islams kann mit Bezug zur Epoche der Spätantike historisch beschrieben werden. Es lässt sich zeigen (vgl. unten), dass der Text des Korans mit jüdischen und christlichen Traditionen in enger Verbindung steht, dass er mit spätantiken christlichen und jüdischen Texten argumentiert und sich von ihnen absetzt. Mohammed verkündete den ihm offenbarten Text anscheinend an Hörer, von denen viele mit christlichem und jüdischem „Bildungsgut“, Erzählungen, Riten und Glaubensinhalten vertraut waren.

Das Weiterleben der Antike in drei Kulturräumen - in Byzanz, im lateinischen Europa und im Islam - setzt die Verbreitung des griechischen Erbes in der Zeit des Hellenismus fort. Die Rezeption und Weiterentwicklung griechischer Wissenschaften, Medizin und Philosophie in Europa durch die Verwendung arabischer Übersetzungen (der griechischen Texte) setzt die lateinische und arabische Welt dabei in eine besondere Beziehung. ${ }^{1}$ Aus dieser Perspektive betrachtet beziehen sich Europa und die arabisch-islamische Welt so eng auf die Traditionen der Alten Welt, dass es nur schwerlich möglich erscheint, eine essentialistische Opposition wie ,jüdisch-christliches Europa versus Koran“ zu vertreten.

Bei einem Blick in die Wissenschaft und deren Rezeption sollte nicht aus dem Blickfeld geraten, dass akademische Diskussionen nicht im luftleeren Raum stattfinden, sondern mit gesellschaftlichen Debatten in Wechselwirkung stehen. Am Beispiel revisionistischer Hypothesen zur islamischen Frühgeschichte und deren breiter Rezeption, lässt sich die Verkettung mit den Sehnsüchten und den Ängsten einer breiteren Öffentlichkeit gut zeigen. So aufschlussreich philologische und historische Perspektiven auf den Koran und den Islam auch sein mögen, für die gegenwärtige Diskussion im Kontext zur Frage der Integration von Muslimen in Deutschland sollte sozialen, ökonomischen, juristischen und politischen Fragestellungen und Lösungsansätzen stets Vorrang zukommen. Vor dem Hintergrund einer etwas unübersichtlichen Situation ist es vielleicht verständlich, dass Menschen in der aktuellen Debatte gerne auf vereinfachende Erklä-

\footnotetext{
${ }^{1}$ Vgl. hier z. B. die Darstellungen von Dimitri Gutas 1998, Franz Rosenthal 1965 und Gotthard Strohmaier 1998.
} 
rungsmuster zurückgreifen und "den Islam“ einem jüdisch-christlichen Europa gegenüberstellen. Der Text des Korans wird dabei häufig selbst, als ob er ein handelnder Akteur sei, als Grund und Ursache der Unvereinbarkeit von Europa und Islam angesetzt.

\section{Auf den Text des Korans reduziert}

Wahrscheinlich wären viele Menschen nahöstlicher Herkunft, die in Deutschland leben, nicht einverstanden, auf ihre Religionszugehörigkeit reduziert zu werden. Auf der individuellen Ebene ist die Zahl derer nicht klein, die sich mehr oder weniger stark durch die religiösen Traditionen ihrer Eltern geprägt oder beeinflusst fühlen. Kulturelle Traditionen türkischer, nigerianischer, iranischer oder chinesischer Muslime sind dabei - wen hätte es gewundert bei dem riesigen geographischen Raum, der gemeinhin als islamische Welt bezeichnet wird - so unterschiedlich, dass sie schwerlich mit einem einheitlichen Islam erklärt werden können. Sicherlich hat die islamische Welt als Kulturraum vieles, was sie verbindet: Der Moscheebau in Westchina und in Westafrika ist strukturell miteinander verwandt, und die in der Architektur verwendete arabische Kalligraphie mit ihren Ornamenten belegt den Bezug zu einem Kulturraum mit unterschiedlichen Ausprägungen auf bildliche Weise. Darstellungen von Menschen und Tieren wird man wahrscheinlich nie in Moscheebauten finden. Auch wenn es kein allgemeines Bilderverbot im Koran oder in der islamischen Tradition gibt, sind nach dem Konsens des islamischen Rechtes Bilder oder Skulpturen in einer Moschee oder an einem anderen Gebetsort nicht vorgesehen. ${ }^{2}$

Wenn über die islamische Welt gesprochen wird, kommt eine Nachlässigkeit der deutschen Sprache ans Tageslicht: Wir sprechen vom Islam, wenn wir einerseits die Religion meinen, aber auch andererseits, wenn wir von der islamischen Welt in ihrer Gesamtheit sprechen. ${ }^{3}$ Zwischen „Christenheit“ für die Gesamtheit der Christen und "Christentum" für die christliche Religion können wir unterscheiden, und auch die Unterscheidung zwischen „Judentum“ und „Judenheit“

\footnotetext{
${ }^{2}$ Vgl. exemplarisch Rudi Paret 1977.

${ }^{3}$ Im Englischen wird hierfür inzwischen der Begriff „islamicate“ verwendet. Im Französischen wird einerseits zwischen „islam“ (kleingeschrieben) zur Bezeichnung der Religion, Gebete, Glaubensinhalte etc. und andererseits „Islam“ (großgeschrieben) für die politischen Strukturen, die Zivilisation, die Kultur etc. der islamischen Welt unterschieden.
} 
ist im Deutschen möglich, aber eher selten. Für den Kulturraum der islamischen Welt, die islamischen Reiche, in denen Christen in manchen Landesteilen die Mehrheit bildeten, die islamischen Wissenschaften oder die islamische Kunst wird der gleiche Begriff verwendet wie für die islamische Religion, die Rechtsauffassungen der Muslime, das Glaubensbekenntnis. Auf diese Unschärfe der Begriffe hat der Islamwissenschaftler und ehemalige preußische Kulturminister Carl Heinrich Becker (1876-1933) hingewiesen, der ganz erheblich dazu beitrug, ein gegenwartsbezogen ausgerichtetes Fach "Islamwissenschaft“ an den deutschen Universitäten einzurichten. Becker verstand den Begriff Islam weit über die religiöse Tradition hinaus als islamische Zivilisation und Kultur, an der ja auch Christen und Juden stark beteiligt waren und die ohne die hellenistische Zeit nicht verstanden werden kann. ${ }^{4}$ Wenn Becker über das griechische Erbe und den Hellenismus schrieb, dass „ohne Alexander den Großen keine islamische Zivilisation" entstanden sei, dann meinte er dies eher im Hinblick auf die Geschichte, Kultur und Gesellschaft, als auf die Religion bezogen. ${ }^{5}$

In der gegenwärtigen Debatte um die Identität Europas fällt auf, dass seit einigen Jahren der Text des Korans sehr präsent ist. Im Frühjahr 2012 verteilten salafistische Gruppen die deutsche Koranübersetzung von Ahmed Rassoul an Passanten in vielen deutschen Städten. ${ }^{6}$ Das Ziel der salafistischen Gruppen ist es dabei, einerseits die Ablehnung der deutschen Rechtsordnung zu inszenieren und für die „wahre Religion“ zu missionieren. In ihrer Inszenierung setzen sie den Text des Korans in den absoluten Mittelpunkt und fordern von der nichtmuslimischen und muslimischen Welt, zum Text des Korans „zurückzukehren“. Die fundamentalistische Ideologie, die zu den Ursprüngen zurück möchte, hat in manchen christlichen Reformbewegungen, z. B. in evangelikalen Gruppen, ihre Entsprechung. So wie Fernsehprediger durch Bibelauslegung, die meist auf den wörtlichen Schriftsinn reduziert ist, die traditionellen kirchlichen Ämter und Rituale außer Kraft setzen möchten, sehen salafistische Gruppen im traditionellen Islam eine aufgrund der über 14 Jahrhunderte gewachsenen Textexegese, des Rechtsverständnisses und beider Verflechtung mit Lebenswelt und Alltag

\footnotetext{
${ }^{4}$ Vgl. Alexander Haridi 2005.

${ }^{5}$ Carl-Heinrich Becker 1910.

${ }^{6}$ Vgl. Muhammad Ahmad Rassoul 2009.
} 
nur noch ein sehr schwaches Echo der ursprünglichen Religion. In ihrer Traditionskritik sind die Salafisten dabei - das wird häufig übersehen, wenn sie als erzkonservativ bezeichnet werden - tief vom Geist der Moderne geprägt. ${ }^{7}$

Als moderne Religionsbewegung fordern sie, um es am Beispiel der heiligen Texte zu veranschaulichen, die absolute Geltung von Koran und Prophetentradition im eineindeutigen Sinne und würden am liebsten das gewachsene traditionelle Textverständnis, das sich in den verschiedenen Räumen der islamischen Welt sehr unterschiedlich ausgeprägt hat, abschaffen. Der Text allein soll gelten. Dass der Wortlaut des Korans bis in die heutige Zeit in verschiedenen Lesarten und Wortlauten (die sich von der Bedeutung her nur sehr wenig unterscheiden) überliefert ist, und dass in Marokko (Lesart Warsch), Sudan (Lesart ad-Dûrî), Tunesien (Lesart Qâlûn), Ägypten und den asiatischen Ländern (Lesart Hafs) leicht divergierende Lesarten des Korans rezitiert werden, ist dem fundamentalistischen Blick dieser Gruppen ein Dorn im Auge. Dass die verschiedenen Rechtsschulen in den 1.200 Jahren sehr unterschiedliche Wege gegangen sind, um die islamische Religion zu interpretieren und dabei Spielräume für Adaptionen, Korrekturen und individuelle Auslegungen zu lassen, ist aus Sicht der Salafisten ein unmoderner Charakterzug des traditionellen Islam. ${ }^{8}$ Die Mehrdeutigkeit, die das intellektuelle und kulturelle Potential des klassischen Islam auszeichnete und ermöglichte, hat Thomas Bauer in einer vor kurzem erschienen Abhandlung sehr eindrücklich beschrieben: ${ }^{9}$ Der Verlust der Ambiguität in der heutigen Welt hat den Islam - etwas überspitzt formuliert - modern gemacht, dadurch aber auch plurale, widersprüchliche, künstlerische Traditionen erstickt.

Der Koran als zentraler Gegenstand der Debatte wird auf der anderen Seite von Islam-Gegnern in Szene gesetzt, wenn, wie im Herbst 2010, evangelikale Christen in Florida den Koran öffentlich verbrennen wollen. Zitate aus dem Koran dienen in zahlreichen Streitgesprächen als wichtige Argumente in der Debatte um Integration und die islamische Religion in Deutschland. Auf einer

\footnotetext{
${ }^{7}$ Vgl. hierzu den Beitrag von Ekkehard Rudolph (S. 191-209) in diesem Sammelband.

8 Ähnlich ist die Vorstellung der Juden von einer Gesetzesordnung, die die Beziehung zwischen Gott und den Menschen in den Mittelpunkt rückt.

${ }^{9}$ Vgl. Thomas Bauer 2011; vgl. auch die beiden Rezensionen in Inamo 2012 von Arno Schmitt und Alexander Flores.
} 
Demonstration der Initiative Pro-Deutschland wird auf einem Transparent gefordert, die „böse“ Verse des Korans zu reformieren (Abb. 1).

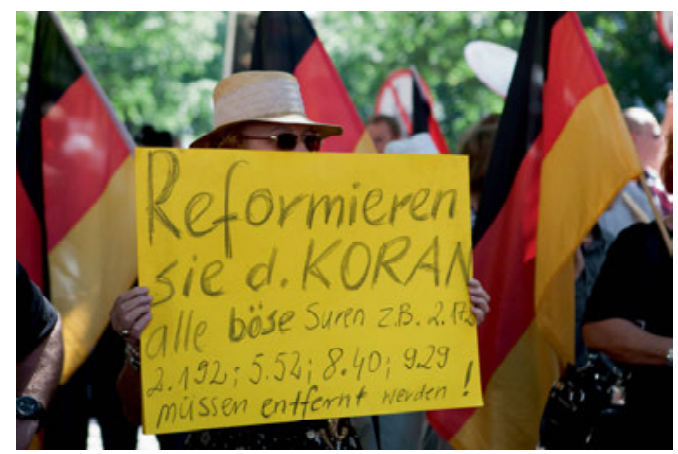

Abb. 1: „Reformiert die bösen Suren“; Transparent einer Pro-Deutschland Demonstration, Berlin, 18.8.2012 (Foto: Thomas Lobenbein)

Bei Protesten im Herbst 2012 gegen den Film „Innocence of Muslims“, der das Leben des Propheten verspottet, werden Koranexemplare von Demonstrierenden gezeigt.

Geht es hier um den Text und die Bedeutung der angegebenen „bösen Suren“? Wirken heilige Texte unmittelbar durch das Handeln ihrer Gläubigen?

Die angegebenen Textpassagen (vgl. Abb. 2) werfen sicherlich Fragen auf. ${ }^{10}$ Wir wissen aus den islamischen Quellen über das Leben des Propheten, dass Mohammed als Oberhaupt des ersten islamischen Gemeinwesens Kriege gegen feindliche Stämme in Arabien geführt hat. Dies mag mit den Vorstellungen von Völkerrecht, moderner Rechtsstaatlichkeit und Menschrechten nicht

\footnotetext{
${ }^{10}$ Die Verszahlen auf dem Transparent scheinen nach der von den Ahmadiyya üblicherweise verwendeten Verszählung gewählt zu sein, bei der die Basmalah (= die am Anfang einer Sure stehende Einleitungsformel „Im Namen Gottes des barmherzigen Erbarmers“) als erster Vers gezählt wird, was bei den anderen Zählsystemen ungewöhnlich ist. Die angegebenen Textpassagen sind deshalb in Abb. 2 wohl nach einer von der Ahmadiyya Gruppe herausgegebenen Übersetzung angegeben. In der deutschen Übersetzung von Hartmut Bobzin, Rudi Paret oder Hans Zirker sind die angegebenen „bösen“ Verse unter Sure 2, Vers 171; Sure 2: Vers 191, Sure 5, Vers 51, Sure 8, Vers 39 und Sure 9 , Vers 29 zu finden (Vers 29 der 9. Sure wird in der Zählung der Ahmadiyya und der üblicherweise verwendeten „kufischen Zählung“ gleich gezählt, da die 9. Sure keine Basmalah hat).
} 


\begin{tabular}{|l|l}
\hline $\begin{array}{l}\text { Textspiegel zu Abb. 1: } \\
\text { Verse des Korans }\end{array}$ & $\begin{array}{l}\text { Deutsche Übersetzung: nach } \\
\text { Der heilige Qurân, Arabisch und Deutsch, Frankfurt 2004. }\end{array}$ \\
\hline Sure 2, Vers 172 & $\begin{array}{l}\text { Und jene, die ungläubig sind, gleichen dem Manne, der das anruft, was } \\
\text { nichts hört als einen Ruf und einen Schrei. Taub, stumm, blind - also } \\
\text { verstehen sie nicht. }\end{array}$ \\
\hline Sure 2, Vers 192 & $\begin{array}{l}\text { Und tötet sie, wo immer ihr auf sie stoßt, und vertreibt sie von dort, von wo } \\
\text { sie euch vertrieben; denn Verfolgung ist ärger als Totschlag. Bekämpft sie } \\
\text { aber nicht bei der Heiligen Moschee, solange sie euch dort nicht angreifen. } \\
\text { Doch wenn sie euch angreifen, dann kämpft wider sie; das ist die } \\
\text { Vergeltung für die Ungläubigen. }\end{array}$ \\
\hline Sure 5, Vers 52 & $\begin{array}{l}\text { Odie ihr glaubt! Nehmet nicht die Juden und die Christen zu Freunden. Sie } \\
\text { sind Freunde gegeneinander. Und wer von euch sie zu Freunden nimmt, der } \\
\text { gehört fürwahr zu ihnen. Wahrlich, Allah weist nicht dem Volk der } \\
\text { Ungerechten den Weg. }\end{array}$ \\
\hline Sure 8, Vers 40 & $\begin{array}{l}\text { Und kämpfet wider sie, bis keine Verfolgung mehr ist und aller Glaube auf } \\
\text { Allah gerichtet ist. Stehen sie jedoch ab, dann, wahrlich, sieht Allah sehr } \\
\text { wohl, was sie tun. }\end{array}$ \\
\hline Sure 9, Vers 29 & $\begin{array}{l}\text { Kämpfet wider diejenigen aus dem Volk der Schrift, die nicht an Allah und } \\
\text { an den Jüngsten Tag glauben und die nicht als unerlaubt erachten, was } \\
\text { Allah und Sein Gesandter als unerlaubt erklärt haben, und die nicht dem } \\
\text { wahren Bekenntnis folgen, bis sie aus freien Stücken den Tribut entrichten } \\
\text { und ihre Unterwerfung anerkennen. }\end{array}$ \\
\hline
\end{tabular}

Abb. 2: Die „bösen Suren“ in ihrem Wortlaut

im Einklang stehen, aber sollen die aufgezählten Zitate - aus ihrem Kontext innerhalb der Suren und aus dem arabischen Kontext ihrer Verkündigung gerissen - unmittelbar für jeden Muslim zu jeder Zeit an jedem Ort gelten? Ist der Koran wirklich das mächtigste Buch der Welt, wie es ein ein Titel des Magazins „Der Spiegel“ formulierte (vgl. Abb. 3)?

Bei den islamisch motivierten Terrorakten der vergangenen Jahre stellt sich die Frage, ob der Korantext hier ursächlich ist und deshalb ggf. einer Revision unterzogen werden soll. Auf andere heilige Texte übertragen ließe sich die gleiche Frage stellen, ob angesichts von Gewaltverherrlichung in der Bibel Textstellen wie das Massaker Simsons an den Philistern aus dem Kanon gestrichen werden müsste: So lesen wir in der Hebräischen Bibel (dem Alten Testament der christlichen Tradition) im Buch Richter 15,15-18: „Und er fand einen frischen Eselskinnbacken. Da streckte er seine Hand aus und nahm ihn und erschlug damit tausend Mann. Und Simson sprach: Mit eines Esels Kinnbacken hab ich sie geschunden; mit eines Esels Kinnbacken hab ich tausend Mann erschlagen. Und als er das gesagt hatte, warf er den Kinnbacken aus seiner Hand, und man nannte die Stätte Ramat-Lehi. Als ihn aber sehr dürstete, rief er den Herrn an 


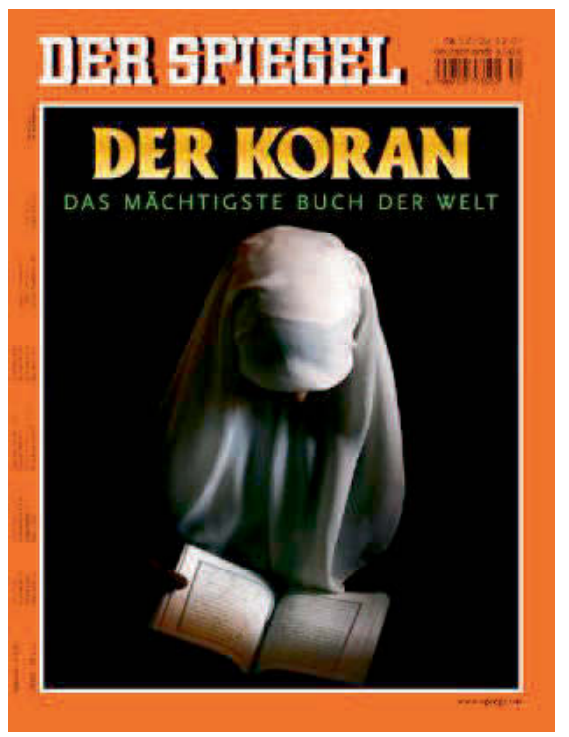

Abb. 3: Spiegel-Titel 24.12.2007, „Der Koran. Das mächtigste Buch der Welt“

und sprach: Du hast solch großes Heil gegeben durch die Hand deines Knechts; nun aber muss ich vor Durst sterben und in die Hände der Unbeschnittenen fallen."11

Auch die Geschlechterrollen in jüdischen und christlichen Texten müssten hinterfragt werden, wie sie z. B. im Wortlaut des jüdischen Morgengebets der Männer zum Ausdruck kommen, das folgendermaßen lautet: „Gelobet seist du, Ewiger, unser Gott, König der Welt, der dem Hahne Erkenntnis gegeben, zu unterscheiden zwischen Tag und Nacht. Gelobet seist du, Ewiger, unser Gott, König der Welt, der mich nicht als Heiden erschaffen. Gelobet seist du, Ewiger, unser Gott, König der Welt, der mich nicht als Sklaven erschaffen. Gelobet seist du, Ewiger, unser Gott, König der Welt, der mich nicht als Weib erschaffen." Der letzte Satz des Morgengebets wird nach dem jüdischen Gebetbuch von Frauen folgendermaßen gelesen: „Gelobet seist du, Ewiger, unser Gott, König der Welt, der mich nach seinem Willen erschaffen."

\footnotetext{
${ }^{11}$ Luther-Übersetzung, revidierte Fassung, Deutsche Bibelgesellschaft Stuttgart 1984.
} 
Wahrscheinlich müssten auch Passagen aus den Briefen des Apostels Paulus revidiert werden, in denen die Rolle der Frau - wen hätte es gewundert - vormodern bestimmt wird. ${ }^{12}$ Neben der Hebräischen Bibel und dem Neuen Testament müssten gegebenenfalls auch abendländische Referenztexte wie Homers Ilias überprüft werden: Sind gewaltverherrlichende Abschnitte der Ilias im Schulunterricht der humanistischen Gymnasien akzeptabel und mit unserer Rechtsordnung kompatibel?

\section{Komplexe Realität - einfache Erklärung: „Der Mythos der Philologie“}

Zurück zu den religiösen Texten und zum Koran, mit dem sich die Wissenschaft in Europa bereits seit 200 Jahren auseinandersetzt: Als das Forschungsvorhaben Corpus Coranicum der Berlin-Brandenburgischen Akademie der Wissenschaften im Jahre 2007 ins Leben gerufen wurde, war das Interesse seitens der Medien nicht gering. Das Vorhaben widmet sich der Grundlagenforschung zum Korantext, es sammelt und dokumentiert: (1) die ältesten Handschriften, (2) Angaben zu den verschiedenen Textvarianten (Lesarten) der islamischen Tradition, (3) Texte aus der Umwelt des Korans und (4) erstellt einen literaturwissenschaftlichen Kommentar. Unter der Überschrift „Ein Buch fehlt“ schrieb Frank Schirrmacher über die eröffnete Frankfurter Buchmesse 2007 und zum angelaufenen Forschungsvorhaben: „Unter all den spirituellen, religiösen und religionsgeschichtlichen Büchern, die die Buchmesse annonciert, fehlt eines, das imstande sein wird, eine Reformation einzuleiten - leider fehlt es auch im Verlag der Weltreligionen. Das Buch, das imstande sein wird, Herrscher zu stürzen und Reiche zu wenden, ist die historisch-kritische Ausgabe des Korans, die in diesem Augenblick an der Berlin-Brandenburgischen Akademie der Wissenschaft vorbereitet wird." ${ }^{\text {13 }}$

\footnotetext{
${ }^{12}$ Z. B. im Brief des heiligen Paulus an die Korinther (1 Kor. 14, 34): „Die Frau schweige in der Gemeinde“ oder im Brief an die Gemeinde von Ephesus (Eph 5, 22): „Die Frau sei dem Manne Untertan“.

${ }^{13}$ Frank Schirrmacher, Frankfurter Allgemeine Zeitung vom 10.10.2007 (am Eröffnungstag der Frankfurter Buchmesse 2007).
} 
Das Akademievorhaben greift eine Idee auf, die bereits der Münchener Arabist Gotthelf Bergsträßer in den 1920er Jahren entwickelt hatte, ${ }^{14}$ den Plan eines Apparatus Criticus zum Koran samt Dokumentation der koranischen Textgeschichte durch ein Fotoarchiv der verfügbaren Koranhandschriften. Bergsträßer und sein Münchener Kollege und Nachfolger Otto Pretzl fotografierten mit einer tragbaren Leica-Fotokamera mehr als 10.000 Handschriftenbilder, die nach dem Tode Pretzls weiterhin in München aufbewahrt wurden. Das Archiv hat glücklicherweise den Zweiten Weltkrieg überstanden und wird vom laufenden Vorhaben an der Berlin-Brandenburgischen Akademie der Wissenschaften ausgewertet. Ein großer Teil der „Koranbilder auf Agfafilm“ ist über die Publikationsseite des Vorhabens einsehbar. ${ }^{15}$ Die Geschichte des nach dem Krieg verlorenen und vor kurzem wiederentdeckten Photoarchivs war dem Wall Street Journal eine Titelgeschichte wert, im Hintergrund sicherlich der Wunsch vieler Leser, dass die historisch-kritische Forschung die "Geheimnisse des Islams" an den Tag bringen könne (vgl. Abb. 4).

Die Auffassung, dass alte Handschriften geheimnisvoll und mächtig sind, scheint ein Teil der modernen Zeit zu sein. Erwartungen dieser Art waren auch in der Diskussion um die Qumran-Fragmente spürbar, als der Fund von Handschriftenfragmenten in Höhlen am Toten Meer für großes Aufsehen sorgte. Die Tatsache, dass das Wall Street Journal, an einem Samstag, einem Tag also ohne Börsengeschäfte, über das „verlorene Gotthelf-Bergsträßer-Filmarchiv“ berichtete, passt in das Denkmuster, den Konflikt zwischen Abendland und Islam als Textkrieg einzuordnen (vgl. Abb. 4).

Gekennzeichnet als Kampf der Kulturen oder Krieg der Religionen, werden Konflikte im Nahen Osten und in Europa, an denen Christen, Muslime und Juden beteiligt sind, isoliert von wirtschaftlichen, sozialen oder politischen Bedingungen wahrgenommen: Am 12. September 2001 kaufte ein Bekannter von mir eine deutsche Übersetzung des Korans, um - wie er es formulierte - zu „verstehen, was da los ist“. Dem protestantischen Grundsatz „sola scriptura“ („durch die Schrift allein“) folgend, ist die Idee der Entschlüsselung des „rät-

\footnotetext{
${ }^{14}$ Vgl. Gotthelf Bergsträßer 1930.

${ }^{15}$ Vgl. Datenbank „Manuscripta Coranica“, abzurufen unter corpuscoranicum.de/handschriften (Betaversion).
} 


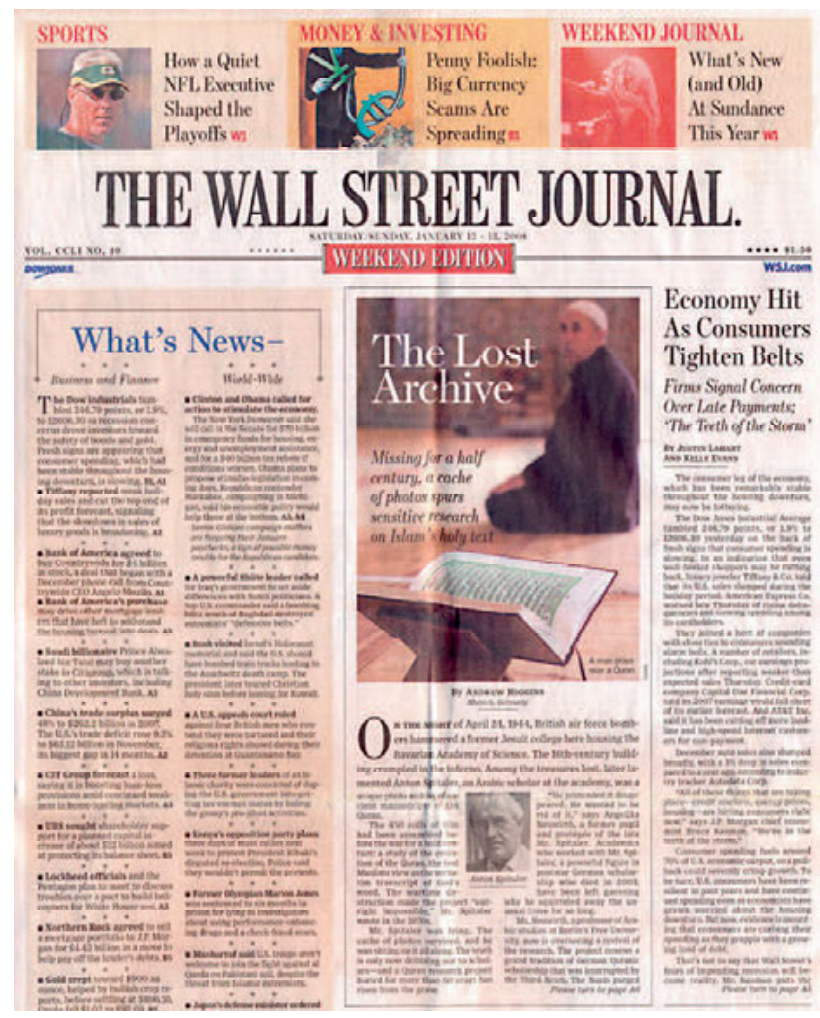

Abb. 4: Andrew Higgins „The lost archive: Missing for a half century, a cache of photos spurs sensitive research on Islam's holy text“ Wall Street Journal 12.01.2008

selhaften Handelns von Muslimen“ durch die Beschäftigung mit den heiligen Texten verlockend. Fast könnte man meinen, dass die Codierung der „Software Muslimische Handlungsmuster“ durch eine genaue Kenntnis des „Heiligen Buches des Islam" vorgenommen werden soll, um es mit einer Metapher zu sagen, die Stefan Weidner vor kurzem verwendet hat. ${ }^{16}$ So oberflächlich es aussehen mag, scheint dies die Wahrnehmung des unmittelbaren Kausalzusammenhangs zwischen „dem, was Muslime tun“ und „dem, was der Koran sagt“, in vielen Fällen zu charakterisieren. Kaum ein Artikel über „die fehlende Aufklärung“ im

\footnotetext{
${ }^{16}$ Vgl. Stefan Weidner 2011.
} 
Islam, ohne auf die fehlende „dringend notwendige“ kritische Ausgabe des Korans hinzuweisen. Fast keine Berichterstattung über Kriege im Nahen Osten und Selbstmordattentate, ohne den Bezug auf islamische Texte und Bilder betender islamischer Frömmigkeit. ${ }^{17}$

Das Bildarchiv alter Koran-Handschriften, das Gotthelf Bergsträßer und Otto Pretzl gesammelt haben, steht für eine ganze Tradition philologischer Koranforschung, die seit dem Zweiten Weltkrieg ein wenig in Vergessenheit geraten ist. Vor zwei Jahrhunderten waren es deutschsprachige Philologen, die die Grundlage für die historisch-kritischen Ansätze zum Koran legten. Abraham Geigers oft zitierte und manchmal missverstandene Promotionsarbeit „Was hat Mohamed aus dem Judenthume aufgenommen?" belegt dies gut. Im Jahre 1833 veröffentlicht, kann Geigers Buch als Meilenstein gesehen werden in der Auseinandersetzung zunächst vornehmlich jüdischer Gelehrter mit den arabisch-islamischen Quellen, die sie neben dem Korpus jüdischer Texte untersuchten. Der Titel von Geigers kleiner Abhandlung ist freilich insofern irreführend, als es dem Autor fern liegt, den Propheten anzugreifen. Er sieht vielmehr in Mohammed eine Art Genie, durch dessen Verkündigung jüdische Texte und Traditionen zu Bestandteilen der muslimischen Tradition geworden seien. Es erscheint dabei geradezu paradox, dass die Gründungsfigur der jüdischen Reformbewegung, der sogenannten „Wissenschaft des Judentums“, die jüdische Tradition bis in den Nahen Osten zurückverfolgt, um das Judentum im post-napoleonischen Mitteleuropa durch Verbindungen zwischen rabbinischen Traditionen und dem Koran neu zu bestimmen. Im Kontext einer christlichen Mehrheitsgesellschaft, die die jüdischen Ghettos aufgelöst hatte, entwickelte eine jüdische Bewegung ihre Vision einer europäisch-jüdischen Identität, indem sie ihre Tradition zu der des Nahen Ostens und der islamischen Religion in Bezug setzte. Der Bezug zum Orient ist in dieser Epoche, also Jahrzehnte vor der zionistischen Bewegung, auch so zu verstehen, dass das jüdische Leben im islamischen Raum positiver bewertet wurde als das im europäischen Mittelalter. ${ }^{18}$

\footnotetext{
${ }^{17}$ Vgl. die Beiträge von Sabine Schiffer (S. 121-137) und Nina Kalwa (S. 139-158) in diesem Sammelband.

${ }^{18}$ Dieser Bezug zum Orient kommt z. B. architektonisch an der Neuen Synagoge in der Oranienburger Straße im Berliner Scheunenviertel zum Ausdruck, die 1866 eingeweiht wurde. Vgl. allgemein dazu Menahem Ben-Sasson 2004.
} 


\section{Wissenschaft in ihrem gesellschaftlichen Zusammenhang}

Mithin sind philologische Ansätze in ihrem gesellschaftlichen Kontext zu sehen. ${ }^{19}$ Durch die Nazizeit und die Vernichtung des Judentums in Europa ist diese Tradition nach dem Zweiten Weltkrieg in Deutschland nicht mehr weitergeführt worden.

Die Bücher und Aufsätze zum Koran und zur islamischen Religion von Gustav Weil, Ignaz Goldziher, Heinrich Speyer, Siegmund Fraenkel, Fritz Shlomo D. Goitein, David Künstlinger, Lazarus Goldschmid u. a. sind bis heute koranwissenschaftliche Referenzarbeiten. Auch Theodor Nöldekes, Gotthelf Bergsräßers und Otto Pretzls Forschungsinteressen am Koran und den klassischen arabischen Texten stehen nicht für sich allein, sondern speisen sich aus der philologischen Tradition, die sich im Zeitgeist des Historismus des 19. Jahrhunderts an deutschsprachigen Universitäten stark etabliert hatte. Ebenfalls ist auffallend, dass es bis zum heutigen Tag viele bedeutende Wissenschaftler auf dem Gebiet der Koranphilologie gibt, die Ordensleute oder Theologen sind.

Zwar hat sich die Erforschung des Korantextes seit der Zeit Abraham Geigers beachtlich entwickelt, dennoch sind sehr grundlegende Fragen zum Koran und der Entstehung der islamischen Religion noch nicht untersucht worden. Bisher gibt es keine historisch-kritische Textausgabe des Korans, und es ist angesichts der vorhandenen Textzeugen in Handschriften und Textvarianten, von denen die islamische Tradition berichtet, unklar, ob sich ein solches Unternehmen umsetzen lässt. Im Rahmen des Potsdamer Akademievorhabens wird seit 2007, unterstützt durch eine Datenbankstruktur, eine systematische Dokumentation der Textmaterialien (Handschriften und Lesarten) aufgebaut, um die zugänglichen notwendigen Materialien zu sammeln und auszuwerten. Im Bereich des Kontextes, also der Umwelt der Verkündigung des Korans, wurde mit dem Zweiten Weltkrieg eine ganze Tradition unterbrochen, und trotz der zahlreichen Publikationen zu syrischen, hebräischen, griechischen Textüberlieferungen und deren Echo im Koran sind wir weit davon entfernt, die Entstehung des islamischen Gründungsdokuments in all seinen Einzelheiten historisch beschreiben zu können.

\footnotetext{
${ }^{19}$ Vgl. z. B. Heinrich Speyers Referenzwerk zu den Biblischen Erzählungen im Koran, das Mitte der 1930er Jahre fertiggestellt wurde und nur heimlich gedruckt werden konnte. Zur Veröffentlichung des Buches wurde das Erscheinungsjahr auf 1931 zurückdatiert, vgl. Franz Rosenthal 2008.
} 
Angesichts zahlreicher unbekannter Größen in einem Forschungsgebiet wundert es nicht, dass in den vergangenen 40 Jahren sehr starke Forschungshypothesen zur Entstehung des Islams und des Korans aufgekommen sind. In den 1970er Jahren vertraten Patricia Crone und Michael Cook, dass die erste islamische Gemeinde und ihr Prophet nicht in Westarabien, sondern im südlichen Palästina entstanden seien. ${ }^{20}$ John Wansbrough veröffentlichte zur gleichen Zeit eine Studie, in der er die Meinung vertrat, der Koran sei erst durch die Koranexegese der klassischen Epoche (8. und 9. Jahrhundert) entstanden, also nicht in Westarabien, sondern in den gelehrten Zentren des Irak. ${ }^{21}$ Ebenfalls in den 1970er Jahren vertrat der Theologe und Islamwissenschaftler Günter Lüling in seiner Studie zum Ur-Koran die Auffassung, dass der ursprüngliche Text des Korans auf eine antitrinitarische christliche Bewegung zurückgehe, und dass der heutige Koran-Text nichts anderes als von der späteren muslimischen Gemeinde verlesene christliche Strophenlieder seien. ${ }^{22}$ Lüling ging davon aus, dass der Urtext z. T. manipuliert und durch andere Setzung diakritischer Punkte verlesen wurde.

Die erwähnten Studien haben keinerlei Handschriften in ihre Untersuchungen einbezogen, über die zugegebenermaßen in den 1970er Jahren wesentlich weniger bekannt war als heute, wo sich der Eindruck stabilisiert, dass wir eine Zahl von kleineren und größeren Koranfragmenten auf Pergament kennen, die ins 7. Jahrhundert, also einige Jahrzehnte nach dem Tod des Propheten Mohammeds, zurückreichen. Zwei Pergamenthandschriften, die ca. die Hälfte des Textes enthalten, werden um das Jahr 700 datiert, eine fast vollständige Handschrift auf Pergament (ms. We II 1913, Staatsbibliothek zu Berlin Preussischer Kulturbesitz) kann auf die Mitte des 8. Jh. datiert werden. ${ }^{23}$

\footnotetext{
${ }^{20}$ Vgl. Patricia Crone und Michael Cook 1977; inzwischen haben sich beide Wissenschaftler von ihren Hypothesen distanziert und haben wenig Zweifel an den großen Rahmendaten der frühislamischen Geschichte. Das Buch „Hagarism“, das sich in der heutigen Zeit sicherlich gut verkaufen würde, ist im Buchhandel nicht mehr erhältlich.

${ }^{21}$ Vgl. John Wansbrough 1977 und 1978.

${ }^{22}$ Vgl. Günter Lüling 1970 und 1974 (Neudruck: 1990, 3. korr. Auflage 2004).

${ }^{23}$ Zur Pariser Handschrift, die um das Jahr 700 datiert wird (BN 328 und den Fragmenten, die in der russischen Nationalbibliothek in St.-Petersburg aufbewahrt werden) vgl. François Déroche 2009. Eine Londoner Handschrift mit 121 Blättern auf Pergament gehört in die gleiche Epoche, vgl. dazu M. Marx, K. Small und A. Fedeli, The Manuscript London or. 2165, Documenta Coranica vol. 1,
} 
Im Bereich der Sprache des Korans und dem religiös-sprachlichen Kontext sind ebenfalls sehr viele grundlegende Fragen ungelöst. Seit dem Zweiten Weltkrieg hat die Zahl von Wissenschaftlern, die sich mit dem Koran beschäftigen und neben arabischen Texten hebräische, syrisch-aramäische Texte auswerten ganz zu schweigen von äthiopischen oder altsüdarabischen Texten -, stark abgenommen. Die Sprache des ältesten arabischen Textes auf Pergament wirft viele Fragen auf, sie entspricht nicht immer den späteren grammatischen Regeln des 8. Jahrhunderts. Bis heute gibt es kein sprachhistorisches und etymologisches Lexikon der arabischen Sprache. Obwohl es zehntausende von inschriftlichen Texten (in altnordarabischen und altsüdarabischen Sprachen aus der Zeit vor dem Islam) neben den Texten der vorislamischen Gedichte gibt, sind diese nie systematisch mit der Geschichte der arabischen Sprache in Beziehung gesetzt worden.

Vor dem Hintergrund solcher Forschungslücken mag es nicht verwundern, dass es breiten Raum für Theorien und Spekulationen gibt. Im Jahre 2000 veröffentlichte z. B. der unter Pseudonym schreibende Philologe Christoph Luxenberg eine Untersuchung zum syrisch-aramäischen Einfluss auf den Text des Korans. ${ }^{24}$ Er untersucht darin ca. 100 Textstellen und vergleicht sie mit der aramäischen Sprache, die er allerdings aus Wörterbüchern belegt und nicht mit konkreten Textbelegen aus der syrischen Literatur. Er kommt bei seiner Studie zu dem Ergebnis, dass der arabische Konsonantentext, der in den ältesten Handschriften nur wenige diakritische Zeichen aufweist, an entscheidenden Stellen verlesen wurde. Da die ältesten Handschriften nicht eindeutig die Konsonanten verzeichnen, haben nach der Hypothese Luxenbergs die arabischen Gelehrten im 8. und 9. Jahrhundert den ursprünglich aramäischen Charakter der Koransprache nicht mehr verstehen können. Der Koran sei ursprünglich sprachlich aramäisch zu lesen, von der religiösen Tradition her handele es sich um einen christlichen Text.

So glaubt Luxenberg zeigen zu können, dass z. B. die Paradiesjungfrauen, den nach dem Verständnis fast aller islamischer Exegeten durch den arabischen Aus-

Leiden 2013 (in Vorbereitung).

${ }^{24}$ Vgl. Christoph Luxenberg 2000. Vgl. unter den zahlreichen Rezensionen die sehr negativen Rezensionen von F. de Blois und S. Hopkins; vgl. seitens Wissenschaftlern aus dem Bereich der syrischaramäischen Sprache die euphorische Rezension von Robert Phenix und Martin Baasten. 
druck „hur 'ain“ (gelegentlich im deutschen Sprachgebrauch „Huris") bezeichnet werden, in der syrisch-aramäischen Vorlage in der Fassung des Urtextes eigentlich Weintrauben seien. In der Literatur der syrischen christlichen Tradition (z. B. bei dem syrischen Kirchenvater Ephrem) wird das Paradies als ein Garten beschrieben, dessen Früchte den auferstandenen Gerechten ewige Freude bereiten. Luxenbergs fast durchweg philologisch-nüchtern formulierte Untersuchung, die für Nichtsprachwissenschaftler bisweilen schwer verständlich ist, wurde bisher nur von sehr wenigen Arabisten akzeptiert. Sie wird sich wahrscheinlich in der Forschung nicht durchsetzen, kann dennoch als wichtiger Beitrag für die Koranwissenschaft gelten. Das Buch hat eine ganze Debatte ausgelöst, zahlreiche Rezensionen und Symposien haben sich mit seiner Hypothese auseinandergesetzt. Auch die Berliner Forschergruppe, aus der das laufende Akademievorhaben „Corpus Coranicum" hervorging, hat in einem Symposium im Jahre 2004 Luxenbergs Hypothese diskutiert. ${ }^{25}$

Im Gegensatz zu den oben erwähnten Thesen aus den 1970er, die lediglich in der akademischen Welt, nicht aber in der breiten Öffentlichkeit diskutiert wurden, hat Luxenbergs Hypothese nach dem 11. September 2001 ein enormes Medienecho erfahren. Zahlreiche deutsche und internationale Presseartikel berichteten über die „syro-aramäische Lesart" und schienen vor allem an dem Beispiel der fehlenden Jungfrauen im Paradies großen Gefallen zu finden. Im Guardian verfasst Ibn Warraq z. B. den Artikel „Virgins what virgins?" ${ }^{26}$ gefolgt von anderen Berichten in DIE ZEIT, Newsweek u. a. ${ }^{27}$ Der Titel des Guardian-Artikels soll eine Gegenfrage auf die fiktive Frage eines ins Paradies einziehenden Gotteskämpfers nach den versprochenen Paradiesjungfrauen sein. Es soll hier nicht bestritten werden, dass die Vorstellung von einem sinnlichen Paradies eine wirkmächtige religiöse Vorstellung für kriegerische Gruppen sein kann, für die Rezeption akademischer Diskussionen hat die Verkettung von Philologie mit tagesaktuellen Ereignissen im Falle der Luxenberg-Hypothese allerdings eine völlig neue Dynamik geschaffen.

\footnotetext{
${ }^{25}$ Vgl. Michael Marx 2003 und 2004 sowie Michael Marx und Nicolai Sinai 2004 (Konferenzbericht).

${ }^{26}$ Vgl. Ibn Warraq in The Guardian vom 12.01.2002.

${ }^{27}$ Vgl. Stefan Theil in Newsweek 27.7.2003.
} 
Eine Gruppe von Islamwissenschaftlern unter der Leitung des Religionswissenschaftlers Karl-Heinz Ohlig (Universität Saarbrücken) hat seit 2005 einen Verein mit dem sprechenden Titel „Inârah“ (wörtlich „Beleuchtung“, gemeint ist „Aufklärung“) gegründet, um einer Gruppe von Forschern einen institutionellen Rahmen zu verleihen. Inârah hat inzwischen sechs Aufsatzsammlungen veröffentlicht, in denen nicht nur weitere Artikel zum „syrisch-aramäischen Urtext“ des Korans erschienen sind, sondern in denen unter anderem die Hypothese vertreten wurde, dass die erste Dynastie der Umayyaden in Wirklichkeit noch Christen gewesen seien. Aufgrund numismatischer Zeugnisse und einer Neuinterpretation mittelpersischer Quellen wird in einigen Artikeln der erwähnten Bände die Hypothese aufgestellt, dass die islamische Bewegung im Ostiran entstanden und dann in Syrien fest etabliert worden sei. Die Person des Propheten Muhammad sei ein historisches Konstrukt der späteren islamischen Tradition (nach dieser Argumentation erst ab dem 8. Jahrhundert geschaffen), um eine mythische Gründungsfigur in der arabischen Halbinsel aufzubauen. Nur wenige Islamwissenschaftler und Arabisten haben diese sehr starken Hypothesen akzeptiert, und auch nicht alle Wissenschaftler in, an und um die „Saarbrücker Schule" scheinen die Hypothese der Nichtexistenz Muhammads und die Entstehung einer islamischen Bewegung im Ostiran zu unterschreiben. Programmatisch gesehen werden die Hypothesen von ganz anderen Ursprüngen, die mit zum Teil philologisch umstrittenen Behauptungen unterlegt werden, mit dem Wunsch verbunden, die islamische Welt zu reformieren bzw. in die angeblich dringend benötigte Epoche der Aufklärung zu versetzen.

Als Günter Lüling seine Thesen vom christlichen Urkoran veröffentlichte, war die Öffentlichkeit nicht daran interessiert. Die englische Übersetzung seines Buches im Jahre 2003 erhielt interessanterweise ebenfalls einen programmatischen Titel: „A challenge to Islam for reformation. The rediscovery and reliable reconstruction of a comprehensive pre-Islamic Christian hymnal hidden in the Koran under earliest Islamic reinterpretations“. Die Vorstellung eines Islams als ursprünglich christliche Strömung scheint verlockend zu sein, gerade vor dem Hintergrund der politischen Ereignisse der letzten zehn Jahre. Unter Titeln wie „Der missverstandene Koran: Warum der Islam neu begründet werden muss“ ${ }^{28}$

\footnotetext{
${ }^{28}$ Vgl. Barbara Köster, Berlin 2010.
} 
„Good Bye Mohammed: Das neue Bild des Islam“, ${ }^{29}$ einem niederländische Titel wie „De omstreden bronnen van het Islam“30 (deutsch „Die umstrittenen Quellen des Islams"), finden die Hypothesen der Saarbrücker Gruppe eine breitere Leserschaft.

Der Publizist Ibn Warraq hat eine große Zahl von Sammelbänden z.T. mit englischen Übersetzungen aus der deutschsprachigen philologischen Forschungsliteratur auf den Markt gebracht, die aus seiner Sicht die Dekonstruktion der islamischen Religion mit philologischen Mitteln beschleunigen können. Die Bände von Ibn Warraq sind trotz ihrer polemischen Ausrichtung insofern verdienstvoll, da sie der englischen Akademia, die inzwischen nur noch wenig Deutsch liest, in Deutsch erschienene philologische Studien von bedeutenden Gelehrten des 19. und 20. Jahrhunderts zugänglich machen.

Das breite Interesse an der Vorstellung, dass Philologie die Welt verändern kann, hat auch in Bestsellern seine Spuren hinterlassen, etwa im Thriller „Terrorist. A Novel“ von 2006. Hierin schildert John Updike den Lebensweg eines Muslims in England, der zum Terroristen wird. An einer Stelle im Roman wird der junge Mann auch mit der Luxenberg-Hypothese von den Weintrauben anstelle der Jungfrauen konfrontiert. Ein anderer Bestseller, „The Last Patriot“ von Brad Thor (erschienen 2008), schickt einen Agenten der CIA auf die Suche nach den ältesten Koranhandschriften, u. a. um die Teile zu erhalten, die - so der Thriller - nach dem Tode des Propheten nicht mehr in den Koran aufgenommen worden sind.

Es gehört zur Entwicklung der Wissenschaften, dass neue Hypothesen diskutiert werden. Im Kontext der gegenwärtigen Debatten in unserer Gesellschaften ist die Philologie offenbar keine unschuldige Größe mehr. Die bisweilen sichtbaren Verknüpfungen von philologischer Hypothese und gesellschaftlichpolitischer Programmatik sind sicherlich nicht auf die erwähnten großen Hypothesen von einem ursprünglich christlichen Korantext oder der historischen Konstruktion des Propheten Mohammed beschränkt. Die wissenschaftliche Beschäftigung mit den grundlegenden Texten der islamischen Religion ist nie neutral erfolgt, eine klare Positionierung ist hier sicherlich hilfreich.

\footnotetext{
${ }^{29}$ Vgl. Norbert G. Pressburg [Pseudonym], Good Bye Mohammed: Wie der Islam wirklich entstand, Norderstedt 2009, in der englischen Übersetzung 2012.

${ }^{30}$ Vgl. Eildert Mulder und Tom Milo 2008.
} 
Wenn Forscher in der Inârah-Gruppe in ihren Veröffentlichungen die Nichtexistenz des Propheten zu belegen versuchen und die „koranische Bewegung“ aus dem östlichen Iran ableiten, dann scheint dies auch für eine breitere Öffentlichkeit interessant zu werden: Das erwähnte populärwissenschaftliche Buch „Good Bye Mohammed: Wie der Islam wirklich entstand“, in dem ein unter Pseudonym schreibender Verfasser die radikalen Thesen zur Islamentstehung in gut lesbarer Weise einer breiteren Öffentlichkeit nahebringt, gefiel dem Ausländerbeauftragten des Landes Thüringen so gut, dass er im September 2010 rund 500 Exemplare an Behörden und Schulen verschickte. Die Versendung der Bücher sollte aus Sicht des Ausländerbeauftragten dazu beitragen, dass sich der Islam endlich mit der Aufklärung beschäftige, was dringend überfällig sei und was die christliche Religion längst hinter sich habe. Hier wird der Versuch der historischen Dekonstruktion einer Religionsgemeinschaft und deren Gründerfigur offenbar mit bestimmten Positionen in der deutschen Integrationspolitik verknüpft. Vielleicht versetzte die Landesregierung den Ausländerbeauftragten deshalb schnell in den vorzeitigen Ruhestand, weil die Versendung der Bücher mit dem Erscheinen von Thilo Sarrazins „Deutschland schafft sich ab“ zeitlich zusammenfiel.

Ein anderes Beispiel für die öffentlich finanzierte Förderung der Verbreitung von in der Orientalistik bisher nicht akzeptierten Hypothesen ist die Aufnahme eines Artikels von Karl-Heinz Ohlig 2007 in das Programm der Bundeszentrale für Politische Bildung, ${ }^{31}$ der vom konstruierten arabischen Propheten und der Entstehung einer Vorform des Islams im Ostiran handelt. Hier haben sich viele Islamwissenschaftler gefragt, weshalb eine in der Orientalistik nicht akzeptierte Hypothese in der Beilage der Zeitschrift „Das Parlament“ als glaubwürdiger Ansatz einem breiten Publikum präsentiert wird. Nichts gegen neue Beobachtungen, Denkanstöße und Hypothesen - sie sind in der wissenschaftlichen Beschäftigung unverzichtbar, auch wenn sie schwach sind.

Die wissenschaftliche Diskussion der Luxenberg-Hypothese hat Positives bewirkt. Vielleicht hat sie sogar die Entstehung des Berliner Langzeitvorhabens, das Angelika Neuwirth, Nicolai Sinai und der Verfasser 2006 konzipierten, beschleunigt. Die Publikationen der Inârah-Gruppen enthalten z. T. hervorragen-

\footnotetext{
${ }^{31}$ Vgl. Karl-Heinz Ohlig 2007.
} 
de Artikel wie die von Gerd und Elisabeth Puin zu den jemenitischen Handschriften. Auch die Aufsätze von Alba Fedeli und Keith Small sind neben einem Aufsatz von Ignaz Goldziher (in erstmalig deutscher Übersetzung aus dem Ungarischen) hier veröffentlicht. Die Artikel der inzwischen erschienenen sechs Sammelbände über die Herkunft des Islams aus Ostiran, die Deutung der umayyadischen Dynastie als christlich-arabische Herrscher und die Hypothese, der Prophet habe nie existiert, ${ }^{32}$ deuten die arabischen, mittelpersischen, syrischen und griechischen Quellentexte aber höchst unzureichend. Die meisten Spezialisten und Lehrstühle auf diesen Gebieten betrachten die vorgelegten Thesen als mangelhaft dokumentiert und in fragwürdiger Weise aus den Quellen abgeleitet. Eine prinzipielle Blockadehaltung in den islamwissenschaftlichen und orientalistischen Wissenschaften ist dafür wohl nicht verantwortlich.

\section{Das Weiterleben der Antike im Islam}

In der Kapitelüberschrift dieses Bandes, in den der vorliegende Beitrag eingeordnet ist, erscheint provokant der Titel des populärwissenschaftlichen Bestsellers von 1960 „Allahs Sonne über dem Abendland“. Die Autorin Sigrid Hunke (19131999) war Mitglied der SS-Abteilung „Ahnenerbe“ und hatte über eine Arbeit zu „Herkunft und Einflüssen fremder Lebensbilder auf den deutschen Menschen“ 1941 promoviert. Ihre Bewunderung für die Araber und die zivilisatorischen Errungenschaften des Islams leiten sich aus ihrer nationalsozialistischen Überzeugung ab und stellen aus ihrer Sicht einen Gegenentwurf zum liberalen Europa dar. Die wissenschaftlichen Errungenschaften, die von den Arabern in der Zeit des europäischen Mittelalters, das aus Hunkes Sicht durch das Christentum kulturell gelähmt war, der europäischen Entwicklung einen entscheidenden Impuls verleiht, stellt sie in ihrem Buch voller naiver Bewunderung dar. Dass sie aus Sicht jener, die zu griechisch-arabischen Übersetzungen und Weiterentwicklungen vielbeachtete wissenschaftliche Arbeiten vorgelegt haben, in ihrer Schwärmerei über das Ziel hinausschießt, ist nicht verwunderlich. Hunke hatte sich mit einem Thema beschäftigt, zu dem ihr offenbar die nötigen philologischen Kenntnisse einfach fehlten.

\footnotetext{
${ }^{32}$ Vgl. Markus Groß und Karl-Heinz Ohlig 2010 und 2012.
} 
Der französische Mediävist Sylvain Gougenheim (Universität Lyon) vertrat 2008 in seinem Buch „Aristoteles auf dem Mont Saint-Michel - Die griechischen Wurzeln des christlichen Abendlandes" die genau umgekehrte These: Dass nämlich die europäische Entwicklung von Medizin, Naturwissenschaften und Philosophie im Hochmittelalter ohne die Übersetzung aus dem Arabischen ins Lateinische verlaufen sei. Europa verdanke den Arabern nach Gougenheims Hypothese also nichts. Seine Darstellung, die eine breite Diskussion in Frankreich ausgelöst hatte und die wissenschaftlich als nicht haltbar bewertet wurde, zeigt, wie sensibel das Thema der europäischen Identität und ihrer Geschichte ist. ${ }^{33}$ Weshalb Gougenheim seine These so massiv formulierte - inzwischen hat er die breite akademische Kritik offenbar teilweise akzeptiert - bleibt, allein auf Grundlage seines Buches zu urteilen, nicht erkennbar. Der Eindruck lässt sich kaum vermeiden, dass der gesellschaftliche Kontext bei Gougenheims „Europaganz-ohne-arabischen-Einfluss-Hypothese" eine maßgebliche Rolle gespielt hat, auch in unserem Nachbarland ist die öffentliche Debatte über den Islam, die Muslime und die europäische Idee nicht neu.

Gerade weil der ins Feld geführten Idee des ,jüdisch-christlichen Abendlandes" starke Bedeutung zukommt, ist der Bereich, den die Bücher von Hunke und Gougenheim berühren, von Bedeutung: Was hat Europa vom Islam übernommen? Aus Sicht der Nationalsozialistin Sigrid Hunke sehr viel: Der arabische Einfluss konnte das aus Hunkes Sicht durch das Christentum gelähmte Europa wiederbeleben. Aus Sicht des Mediävisten Sylvain Gougenheim, der nach den Debatten um sein Buch Fehler und Ungenauigkeiten eingeräumt hat, verdanken wir den Arabern fast nichts: Griechische Wissenschaft und Philosophie konnten nach Gougenheims Hypothese durch Kontakte zur byzantinischen Tradition in die Zentren der europäischen Gelehrsamkeit gelangen, auf die Araber war Europa nicht angewiesen.

Der arabische Beitrag zur Entwicklung Europas ist in der Tat nicht gering, nicht nur auf dem Gebiet der Philosophie, und er beschränkt sich mit Bewässerungstechniken in Spanien, der Verwendung von Spitzbögen in der Architektur der Gotik und der Übernahme der indischen Zahlen (die wir aufgrund ihrer Vermittlung ,arabisch“ nennen) nicht auf den Bereich zahlenmäßig kleiner aka-

\footnotetext{
${ }^{33}$ Vgl. Roger Pol Droit, „Et si l’Europe ne devait pas ses savoirs à l'islam ?“, Le Monde 4.4.2008.
} 
demischer Gruppen. Im Bereich der Medizin ist hier der „Medizinische Kanon“ (arab. „al-Qanun fi at-tibb“) des Avicenna (sein arabischer Name lautete Abu Ali al-Husain ibn Abdallah ibn Sina, 980-1037) zu nennen, der im 12. Jahrhundert von Gerhard von Cremona ins Lateinische übersetzt wurde und bis in die Neuzeit als medizinwissenschaftliche Referenz galt. Zeitgleich wurde in Toledo Avicennas philosophisches Werk „Kitab ash-shifa“ (wörtl. arab. „Buch der Heilung") übersetzt, das Themen aus dem Bereich der Astronomie, Geometrie, Mathematik, Philosophie und Musik behandelt.

Neben Avicenna ist Rhazes (Abu Bakr Muhammad ibn Zakariya ar-Razi, gest. ca. 930) als einer der bedeutenden Gelehrten in der islamischen Welt zu nennen, die griechische Wissenschaften der Antike rezipiert und weitergeführt haben. Er verwandelte die Lehre des griechischen Mediziners Galen (2. Jahrhundert) in ein Curriculum der Medizinhochschulen. Er soll bereits mit Experimenten gearbeitet und Krankheiten wie Pocken und Masern beschrieben haben. Wie Abb. 5 anschaulich zeigt, hat auch der aus Ray (südlich des heutigen Teheran gelegen) stammende Gelehrte durch Übersetzungen seiner Werke seinen Platz in einer europäischen Universalgeschichte gefunden.

Der erwähnte Übersetzer Gerhard von Cremona übersetzte nicht nur Schriften von Avicenna und Rhazes, sondern auch das Algebra-Lehrbuch des AlKhwarizmi (von dessen Name das Wort Algorithmus sich ableitet). Auch der Almagest, das astronomische Hauptwerk des Claudius Ptolemäus (2. Jh. n. Chr.), wurde vom Arabischen ins Lateinische übersetzt. Auf der Grundlage der lateinischen Übersetzung (aus dem Arabischen) des Almagest entwickelte Nicolaus Kopernikus die Grundlage des heliozentrischen Weltbildes.

Die wenigen Beispiele sollen genügen, um darauf hinzuweisen, dass die Aufnahme der für Europa so bedeutenden Epoche der Antike neben der direkten Rezeption aus dem Griechischen, die ja nie vollständig ausgefallen ist, oft über das Arabische als Wissenschaftssprache der islamischen Welt gelaufen ist. Neben dem Almagest hatte der Übersetzer Hunain ibn Ishaq (gest. ca. 873) in Bagdad eine überaus große Zahl griechischer Werke, z. T. aus syrischen Fassungen, ins Arabische übersetzt, darunter offenbar auch Platons Staat, der uns allerdings nicht erhalten ist. Das Weiterleben der Antike im Islam hatte seinen Schwerpunkt auf dem Gebiet der Medizin, der Philosophie (vor allem die Schriften des 

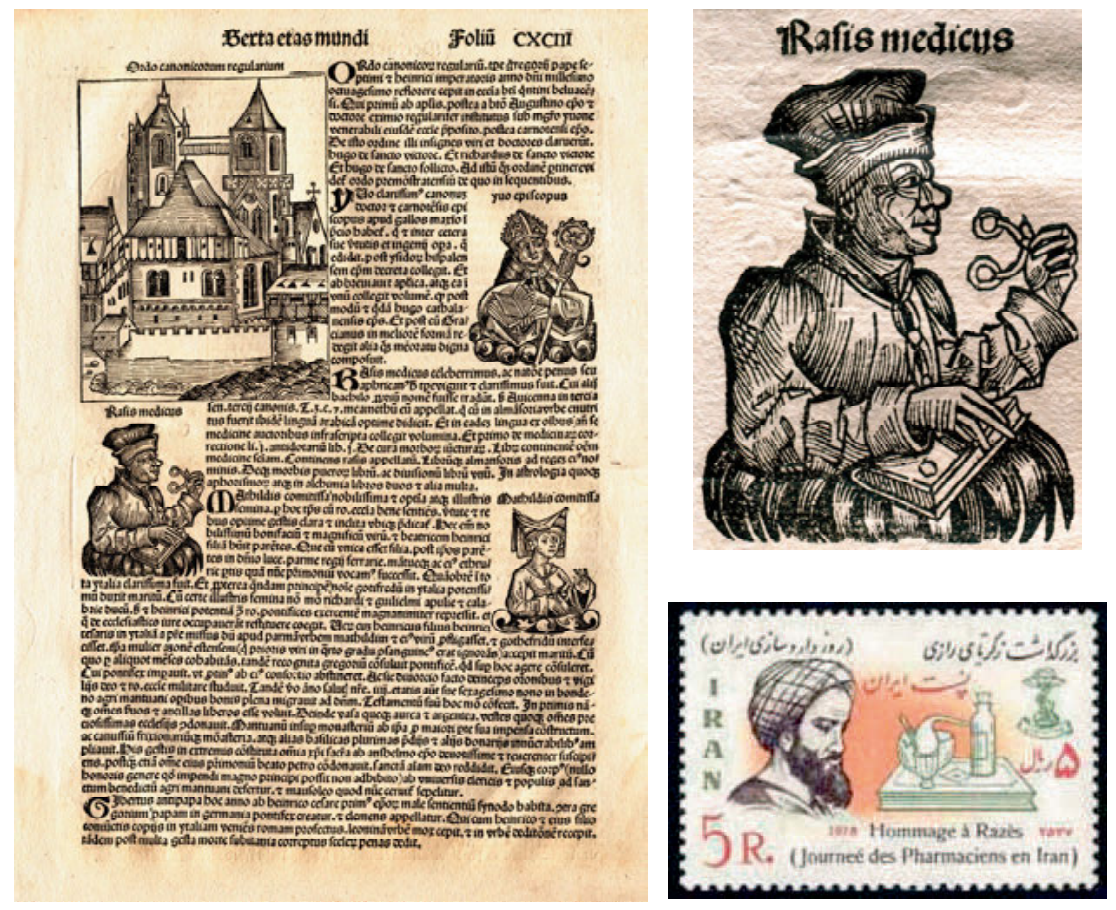

Abb. 5: Links: Der aus Ray (südlich von Teheran) stammende Arzt Rhazes (gest. ca. 930) in einem Holzschnitt von M. Wolgemut zu H. Schedels Weltchronik (1493). Rechts oben: Ausschnittvergrößerung; rechts unten: Gedenkbriefmarke Iran 1978.

Aristoteles), der Mathematik, der Optik, der Astronomie, der Erdvermessung u. a. Eine arabische Übersetzung von Homers Ilias oder Odyssee, der Tragödien und Komödien hat uns nicht erreicht. Die Rezeption der Antike in den griechischen Wissenschaften hat ihren eigenen Charakter und spezifische Interessenschwerpunkte gesetzt. Es mag stimmen, dass „griechische Wissenschaft“ und „islamische Gelehrsamkeit“ wenig miteinander kommunizierten. Die Zahl der islamischen Religionsgelehrten, die Griechisch lernen, war und ist auch heute noch eher klein. Es war sicher kein Einzelfall, dass Gelehrte wie al-Razi mit dem religiösen Establishment aneinander gerieten: Im Falle des Rhazes so stark, dass 
er seine Position in Bagdad aufgab und in seine Heimatstadt Ray zurückkehrte. Es ist richtig, dass viele Übersetzer und Gelehrte Christen waren, so z. B. der Übersetzer Hunain ibn Ishaq, der in der Hauptstadt des abbasidischen Reiches lebte. Als Christ wären ihm politische Positionen vorbehalten geblieben. Ihn und andere Nicht-Muslime nur unter dem Vorzeichen von Unrecht und Unterdrückung zu sehen, wird den intellektuellen Errungenschaften der islamischen Welt nicht gerecht.

\section{Der Koran und die Spätantike}

In der modernen Epoche wird der Beitrag der christlichen oder jüdischen Gelehrten an den Errungenschaften der islamischen Zivilisation oft ausgeblendet, zum Teil aus politischen Gründen: Mit dem jüdischen Philosophen Maimonides (geb. in Cordoba ca. 1035, gest. in Kairo 1204) als Teil der Geschichte des islamischen Ägyptens tun sich Æ̈gypter in der heutigen Zeit manchmal schwer. Dass Gregorius Bar Hebräus (ca. 1225-1286), ein auf Syrisch und Arabisch schreibender Kirchenlehrer und Philosoph, im islamischen Raum wirkte, fehlt in manchen Darstellungen der islamischen Geschichte. Das Verständnis von Islam als Konfession und Religion hat heute, grob formuliert, das Verständnis von Islam als islamischem Kulturraum oder islamisch geprägter Welt verdrängt. Manche Darstellungen des griechischen Erbes im Islam gehen soweit, dass sie das Weiterleben und die Fortführung der griechischen Antike gänzlich als Leistung muslimischer Gelehrter verstehen. ${ }^{34}$

Wenn wir zum Text des heiligen Buches der Muslime kommen, wird die Frage des Bezugs zur Antike auf eine ganz andere Weise bedeutungsvoll. Der Islam, der sich mit den Reichen der Umayyaden und Abbasiden im Nahen Osten etablierte, blieb die Religion der politischen Elite, die allerdings zahlenmäßig in Ägypten, Syrien und im Irak über Jahrhunderte hinweg eine Minderheit darstellte. Der Text des Korans, seine Exegese, die Lexikographie und die Grammatik der arabischen Sprache etablierten sich in einer Welt, in der Christen und Juden präsent waren und auch nach dem Verständnis der islamischen Jurisprudenz gesellschaftliche und religiöse Rechte behielten. Vielleicht trug die Präsenz

\footnotetext{
${ }^{34}$ Vgl. Gotthard Strohmaiers Artikel „Europa im schrägen Blick eines Gräkoarabisten“, in G. Strohmaier 2012.
} 
der Christen, Juden und Zoroastrier dazu bei, dass die relativ neuentstandene islamische Gelehrsamkeit das Bedürfnis hatte, sich von ihnen abzugrenzen. Im Curriculum der islamischen Hochschulen, bei denen das islamische Recht den Kernbereich darstellt, kamen griechische Wissenschaften jedenfalls kaum vor. Der Islam verstand sich als die letzte Offenbarungsreligion, in der die christliche und die jüdische Religion weitergeführt und von theologischen Fehlern wie der Trinitätslehre oder den aus islamischer Sicht übertriebenen jüdischen Rechtsvorstellungen befreit wurde. Christen und Juden haben aus islamischer Sicht zwar durch ihre Propheten Anteil an der göttlichen Offenbarung, haben sich aber durch Übermittlungsfehler ihrer Text- und Exegesetraditionen von der „ursprünglichen monotheistischen Lehre" entfernt.

Das Studium des Korans, den man sich als Verschriftlichung der Verkündigungen des Propheten vorstellen kann, ist in der islamischen Gelehrsamkeit in ein eigenes Curriculum von Disziplinen eingebettet, bei denen der Wortlaut des Textes mit Referenzwerken der arabischen Grammatik, Lexikographie und Rhetorik studiert wird. Interessanterweise hat die islamische Wissenschaft ein eigenes Konzept einer historischen Entwicklung innerhalb des Korantextes entwickelt: die Wissenschaft von den „Asbab nuzul", von den „Gründen der Offenbarung “. Für den Text selbst kennt der traditionelle Islam verschiedene Textüberlieferungen des koranischen Wortlautes. Die Vorstellung von einem im Wortlaut festgelegten Gotteswort, das direkt zwischen zwei Buchdeckel gelangt ist, ist keine traditionell islamische Vorstellung, das Dogma einer Verbalinspiration kennt der klassische Islam nur in einer Untergruppe der hanbalitischen Rechtsschule. ${ }^{35}$ Auch das islamische Recht leitet sich nicht unmittelbar aus Koran und Hadithe ab. Wie z. B. Harald Motzki zeigen konnte, war es eine bestimmte regionale Praxis von Rechtsprechung, die den Ausgangspunkt des islamischen Rechts bildet. ${ }^{36}$ In der späteren Entwicklung wurden die eigentlich religiösen Texte, z. B. die Hadithe, immer stärker in die juristischen Argumentationen aufgenommen, vielleicht auch um dem islamischen Recht eine stärkere islamische Farbe zu geben. Die geringe Bedeutung des Korantextes im islamischen Recht ist nicht verwunderlich, da der Text nur wenige rechtliche Bestimmungen enthält, vom

\footnotetext{
${ }^{35}$ Vgl. Joseph van Ess 1996.

${ }^{36}$ Vgl. Harald Motzki 2002.
} 
Textvolumen her ca. $5 \%$ (der insgesamt ca. 6.300 Verse). Als juristischer Kodex ist der Text des Korans ungeeignet, historisch hatte der Text diese Funktion nie.

Auf dem Gebiet der Grundlagenforschung bleiben jedoch - im Vergleich zur Entstehung des Neuen Testaments, dessen historisch-kritische Erforschung seit langer Zeit etabliert worden ist -, viele Fragen offen. Ob eine kritische Edition nach dem Vorbild anderer kritischer Textausgaben auf Grundlage der Koranhandschriften und der Textvarianten in den Lesarten erstellt werden kann, bleibt im Augenblick noch offen. Verglichen mit der Textgeschichte des Neuen Testaments liegen für die koranische Textgeschichte mit ca. 1.200 Blättern aus Pergament-Handschriften - neben Fragmenten auf Papyrus und Inschriften, z. T. vor 750 v. Chr. - erstaunlich frühe Textzeugen vor.

Für den Bezug zu europäischen Traditionen ist das Studium der Umwelt des Textes von großem Interesse. In diesem Bereich werden in der Potsdamer Arbeitsstelle „Texte aus der Umwelt des Korans“ aus syrischen, hebräischen, altarabischen, griechischen u. a. Quellen in Form einer Datenbank aufgearbeitet. Eine erste Anzahl ist über die Publikationsseite des Vorhabens einsehbar, das Unternehmen selbst steckt noch in den Anfängen. Es ist uns dabei wichtig, Belegstellen in ihrem Zusammenhang auszuwerten und für das Studium der kulturellen und religiösen Umgebung zu erschließen. Wenn beispielsweise in der 3. Sure Jesus Christus aus Lehmfiguren lebendige Vögel schaffen kann (Abb. 6), dann wurde dies häufig zum Beleg einer Copy-Paste-Methode des koranischen Textes genommen: Im gegebenen Fall sei das Vogelwunder des Thomas-Kindheitsevangeliums in den Korantext hineinkopiert worden. ${ }^{37}$

Vergleicht man die Texte genau, dann wird deutlich, dass es neben Übereinstimmungen theologische Unterschiede gibt: Christus belebt im Koran die Lehmfiguren nicht aus eigener Kraft, sondern tut dies auf Geheiß Gottes. Wenn wir uns die apokryphe Tradition als eine Lehrgeschichte der frühen Christen zur Göttlichkeit Christi vorstellen, der bereits als Kind wie Gott Lebewesen schaffen konnte, dann setzt hier der Text des Korans mit einer Argumentation an, nach welcher es der Prophet Jesus war, der durch die Erlaubnis Gottes das Wunder vollbringen konnte. Ebenso wie Jesus im gleichen Koranvers Heilungen durchführen und Tote erwecken konnte - nur durch die Kraft Gottes, die ihm zukam.

\footnotetext{
${ }^{37}$ Vgl. z. B. William St. Clair Tisdall 1911.
} 


\begin{tabular}{|c|c|c|c|}
\hline \multicolumn{4}{|c|}{ Das Vogelwunder Christi im Kindheitsevangelium des Thomas und im Koran } \\
\hline \multicolumn{2}{|c|}{ Kindheitsevangelium des Thomas } & \multicolumn{2}{|c|}{ Koran - Sure 3, Vers 49 (Übers. H. Bobzin) } \\
\hline 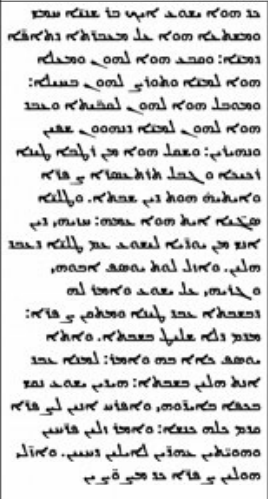 & 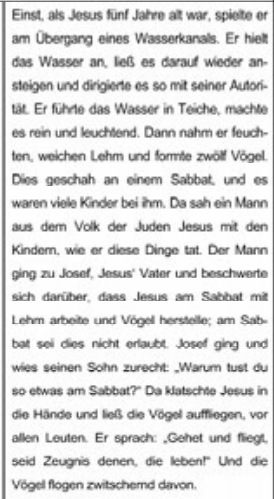 & 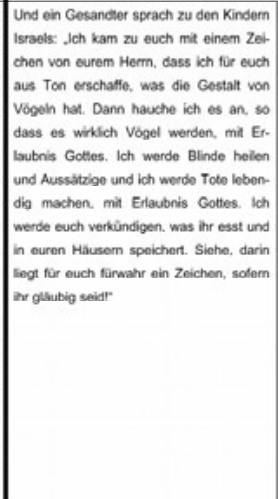 & 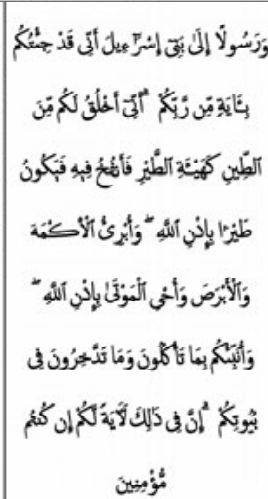 \\
\hline \multicolumn{4}{|c|}{ 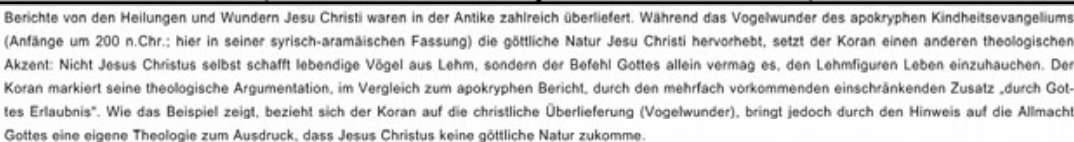 } \\
\hline
\end{tabular}

Abb. 6: Das „koranische Vogelwunder“ im Vergleich mit der apokryphen Überlieferung.

Wir sehen an dieser Stelle, wie eine Lehrerzählung des spätantiken Christentums aufgegriffen wird, dabei aber in eine ganz andere theologische Richtung gelesen wird. Der Nachweis jüdischer oder christlicher Traditionen in der Umwelt des Textes ist auch bei der Deutung von Sure 112 wichtig. Das jüdische Gebet, das Schma Yisrael, scheint im Vers 1 der Sure ein Echo zu finden, allerdings nicht auf die jüdische Gemeinde allein bezogen, sondern universal formuliert. Das christliche Glaubensbekenntnis, das Menschen in der Umgebung des Propheten kannten, könnte in seiner Verneinung in den Versen 2 bis 4 der 112. Sure ein Echo gefunden haben (Abb. 7).

Beweisen lassen sich solche Zusammenhänge strenggenommen nicht, aber die Ähnlichkeiten und Differenzen zwischen Korantexten und religiösen Texten der Spätantike können einen Schlüssel zum historischen Verständnis des Textes bringen. ${ }^{38}$ Die Negierung eines zeugenden Gottes könnte sich natürlich auch

\footnotetext{
${ }^{38}$ Vgl. z. B. die von David Kiltz und Yousef Kouriyhe ausgewerteten syrisch-aramäischen Textzeugen aus der vorislamischen Zeit zum Verständnis der koranischen Eschatologie und Jenseitsvorstellungen, Datenbank „Texte aus der Umwelt des Korans“ abrufbar unter corpuscoranicum.de (Betaversion).
} 


\begin{tabular}{|c|c|c|c|c|c|}
\hline \multicolumn{2}{|c|}{$\begin{array}{l}\text { Nicăno-Konstantinopolitanisches } \\
\quad \text { Glaubensbekenntnis } \\
\text { (Konzil von Konstantinopel, } 381 \text { n. Chr.) }\end{array}$} & \multicolumn{2}{|c|}{$\begin{array}{l}\text { Jüdisches Gebet .Sch'ma Jisrael" } \\
\text { 5. Buch Mose 6,4 }\end{array}$} & \multicolumn{2}{|c|}{ Koran-Sure 112} \\
\hline Obers. & Grechisch & Obers. & Hebraisch & Obers, (H. Bobzin) & Arabisch \\
\hline $\begin{array}{l}\text { Wir glauben an einen } \\
\text { Gott, }\end{array}$ & 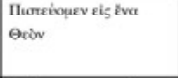 & $\begin{array}{l}\text { Höre Israel! Der Herr ist } \\
\text { unser Gott, der Herr ist } \\
\text { einer. }\end{array}$ & 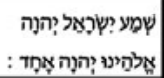 & $\begin{array}{l}\text { Sprich: ,Er ist Gott, der } \\
\text { Eine. }\end{array}$ & 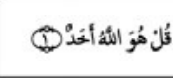 \\
\hline $\begin{array}{l}\text { den Vater, den } \\
\text { Allmåchtigen, der alles } \\
\text { geschaffen hat, Himmel } \\
\text { und Erde, den Schōpler } \\
\text { alles Sichtbaren und } \\
\text { Unsichtbaren. }\end{array}$ & 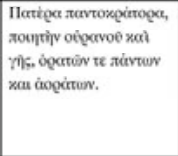 & & & Gott, der Beständige, & اللَّ الصَهْدُد (5) \\
\hline $\begin{array}{l}\text { Und an den einen Herrn } \\
\text { Jesus Christus, den } \\
\text { Sohn Gottes, der als } \\
\text { Einziggeborener aus } \\
\text { dem Vater gezeugt ist } \\
\text { vor aller Zeit, Licht aus } \\
\text { Licht, wahrer Gott aus } \\
\text { wahrem Gott, gezeugt, } \\
\text { nicht geschaffen, } \\
\end{array}$ & 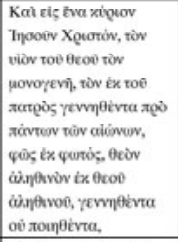 & & & $\begin{array}{l}\text { Er zeugte nicht und } \\
\text { wurde nicht gezeugt. }\end{array}$ & 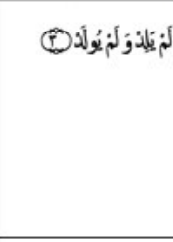 \\
\hline $\begin{array}{l}\text { eines Wesens mit dem } \\
\text { Vater: }\end{array}$ & 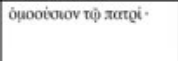 & & & $\begin{array}{l}\text { Und keiner ist ihm } \\
\text { ebenbürtig." }\end{array}$ & 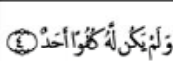 \\
\hline
\end{tabular}

Abb. 7: Sure 112 im Vergleich mit dem jüdischen Schma-Yisrael und dem Nizäno-Konstantinopolitanischen Glaubensbekenntnis.

auf die altarabischen Vorstellungen beziehen, die uns in inschriftlichen (= epigraphischen) Texten Arabiens überliefert sind und denen zufolge Gott „Töchter“ hat. ${ }^{39}$ Die auffallend starke Ähnlichkeit mit dem jüdischen Schma-Yisrael-Gebet und mit dem christlichen Glaubensbekenntnis belegt ganz gut, dass offenbar vom Koran angesprochene Adressaten oder Zuhörer mit jüdischen und christlichen Inhalten vertraut waren. Fast wörtlich greift der 3. und 4. Vers der 112. Sure die Formulierung des Nizäums auf und negiert sie. Der Text des Korans dokumentiert in seiner Formulierung gewissermaßen jüdische und christliche Präsenz.

Bei solchen Überlegungen soll der geographische Kontext nicht aus dem Blick geraten: Mekka und Medina, in denen der Text verkündet worden war, sind nicht weit entfernt vom himjaritischen Reich im Jemen. Anfang des 6. Jh. wurde das Judentum durch den König Dhu Nuwas einige Jahre zur offiziellen Religion des himjaritischen Reiches erhoben.

${ }^{39} \mathrm{Zu}$ den in den südarabischen Inschriften erwähnten „Töchtern Gottes“vgl. Christian Robin 2007. 
Im Jahre 525 wurde durch die militärische Intervention der Äthiopier das Christentum zur offiziellen Religion. Vielleicht wird die archäologische Forschung hier weitere Materialien ans Licht bringen, wie es die Ausstellung „Roads of Arabia" in Berlin (Januar bis März 2012) eindrucksvoll gezeigt hat. Die Geschichte des vorislamischen Arabiens hat offenbar einen stärkeren Bezug zur Antike als angenommen: So wurde z. B. eine offizielle römische Inschrift aus dem späten 2. Jahrhundert $n$. Chr. entdeckt, die belegt, dass die Stadt Hegra (das heutige Mada'in Saleh in Saudi-Arabien) Teil des römischen Reiches war. ${ }^{40}$

Aus Qaryat al-Faw im Südwesten des saudi-arabischen Königreiches wurden eine Bronzebüste (Abb. 8) und im römischen Stil gemalte Fresken ausgegraben, die an die Kunst im römischen Reich erinnern. Die Epoche der Spätantike, die uns aufschlussreiches Material zur Rekonstruktion der kulturellen Umgebung des Textes liefert, muss also sehr breit angesetzt werden.
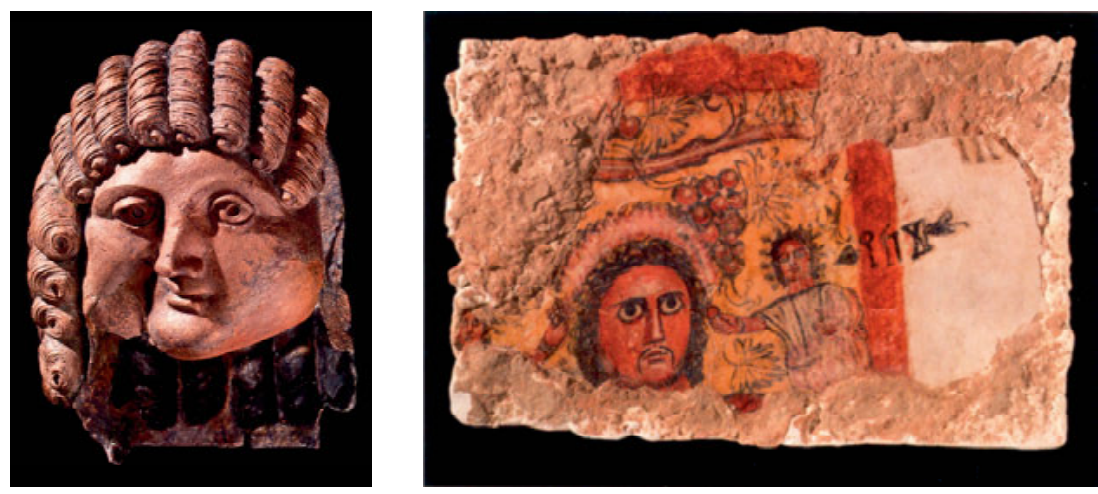

Abb. 8: Bronzebüste und Wandfresko aus Qaryat al-Faw (Saudi Arabien), ca. 1./2. Jh. n. Chr. (vgl. Ausstellungskatalog "Roads of Arabia“).

Wir sehen, dass dem Kontext des Korans eine Schlüsselrolle zukommt und dass wir uns hier in einem kaum zu überblickenden Gebiet befinden. Angelika Neuwirth hat in ihrem programmatischen Band „Der Koran als Text der Spätantike" (2010) insbesondere die Bezüge des Textes zu jüdischen und christlichen Traditionen untersucht und den starken Bezug des Korans zur jüdischen und

\footnotetext{
${ }^{40}$ Vgl. Dhaifallah al-Talhi und Mohammad al-Daire 2005.
} 
christlichen Spätantike herausgearbeitet. Über diesen Bereich der theologischen Traditionen der Christen und Juden hinausgehend müsste man auch die Spätantike des sassanidischen (iranischen) Reiches einbeziehen, in dessen politischen Einzugsbereich Mekka seit Ende des 6. Jahrhunderts lag. Vor allem aber die antiken Zivilisationen Südarabiens, die mehr als ein Jahrtausend bis kurz vor die Zeit Mohammeds Bestand hatten, müssten systematisch zum Verständnis der frühen islamischen Geschichte ausgewertet werden. Dies ist bisher jedoch nie geschehen.

Für die historische Sichtweise auf den Text ist der Gedanke wichtig, dass die 114 Suren des Korans in eine chronologische Reihenfolge zu setzen sind, die sich von ihrer Anordnung im Buch unterscheidet. Hier hatte Theodor Nöldeke (1834-1930), aufbauend auf den Angaben der islamischen Tradition und eigener stilistisch-sprachlicher Kriterien, eine chronologische Reihenfolge vorgeschlagen, die in vier Phasen (Mekka 1, Mekka 2, Mekka 3 und Medina) die Themen und die Aussagen der Suren in eine historische Abfolge stellt.

Angelika Neuwirth hat 1982 in ihrer Habilitationsschrift aufbauend auf Nöldekes "Geschichte des Qorans" (1860) durch literaturwissenschaftliche Methoden die mekkanischen Suren genauer untersucht und damit Grundlagen für weitere philologische Studien bereitet. Sie konnte aufgrund formkritischer Analysen Strukturen nachweisen, die für innertextliche Entwicklungslinien sprechen Diese Arbeiten, die sich dem Text in der geschichtlichen Abfolge seiner Verkündigung zwischen den Jahren 610 und 632 widmen, stützen sich auf den Textbestand, wie die islamische Tradition ihn vermittelt; die Untersuchungen machen dabei deutlich, wie etwa Nicolai Sinai gezeigt hat, dass sich historische Entwicklungen aufzeigen lassen, die schwerlich in einem vollkommen unverstandenen und schlecht überlieferten Text auszumachen wären. ${ }^{41}$

Neuwirths Ansatz, den sie ausführlich in ihrer Monographie „Der Koran als Text der Spätantike“ (2010) erläutert und ihrer Surenkommentierung zugrunde legt, sieht den Text des Korans als eine Art Niederschrift eines Kommunikationsprozesses zwischen dem Verkünder und seinen Zuhörern, die sowohl aus denen bestehen, die später die Gemeinde bilden, als auch aus den Gegnern des Verkünders. Wenn wir uns, wie die oben genannten Beispiele belegen sollten,

\footnotetext{
${ }^{41}$ Vgl. Nicolai Sinai 2009.
} 
die Umwelt als antik oder spätantik gebildet vorstellen, dann können wir den Versuch unternehmen, die Diskurse des Korantextes in seiner historischen Entwicklung nachzuzeichnen. Neuwirth hat inzwischen einen Kommentarband zu den frühmekkanischen Suren (Mekka-1-Periode) veröffentlicht und dabei starke Bezüge der frühen Suren zu den Texten der Psalmen herausgearbeitet. ${ }^{42}$

Aufbauend auf Neuwirths Hypothese des Kommunikationsprozesses, hat Nicolai Sinai im Rahmen des Akademievorhabens "Corpus Coranicum" einen chronologisch-literaturwissenschaftlichen Kommentar zu den frühmekkanischen Suren erarbeitet, z. T. mit leicht divergierenden Einzelanalysen und abweichenden formalen Kategorisierungen, der online veröffentlicht ist. ${ }^{43}$

Beide Kommentare belegen, dass der überlieferte Text historisch zugänglich ist und dass der Koran nicht notwendig dunkel und unverständlich bleibt, sondern dass sich entwickelnde Argumentationslinien nachgewiesen werden können.

Sicherlich sind auch hier die vorgelegten Arbeiten weit davon entfernt, endgültige Ergebnisse zu liefern. An der Potsdamer Arbeitsstelle wird bewusst der dokumentarischen Ebene Vorrang eingeräumt, um in einem Bereich historische Forschung zu ermöglichen, in dem grundlegende Materialien wie die vorhandenen Handschriften, Lesarten, Umwelttexte u. a. noch unzureichend erschlossen sind.

\section{Ausblick: Bitte kein Kulturkampf!}

In den europäischen Gesellschaften findet eine breite Diskussion zur Präsenz des Islams statt, die über das Alltagsgeschäft der Konflikte des Miteinander- oder Nebeneinanderlebens in die Bereiche der historischen, philologischen und kulturellen Fragen hineingreift. Wenn das vermeintlich jüdisch-christliche Europa dabei dem „unmodernen“ Islam gegenübergestellt wird, drängt sich der Eindruck eines Kulturkampfes auf. Die Metapher „Software Islam“, die die Muslime angeblich kontrolliert, erinnert an das Klischee von den ultramontanen (heute würde man sagen: „ferngesteuerten“) Katholiken, die in den 70er Jahren des

\footnotetext{
${ }^{42}$ Vgl. Angelika Neuwirth, Der Koran: Bd. 1: Frühmekkanische Suren. Poetische Prophetie, Handkommentar mit Übersetzung von Angelika Neuwirth, Frankfurt/Berlin 2012.

${ }^{43}$ Online zugänglich unter corpuscoranicum.de in Betaversion.
} 
19. Jahrhundert - in der Zeit des sogenannten Kulturkampfes - als nicht loyale Staatsbürger gesehen wurden. Auch hier zeigte sich eine symbolische Ebene, wenn die katholische Bevölkerung im Rheinland, das nach der napoleonischen Zeit von Preußen regiert wurde, das Bedürfnis hatte, z. B. Wallfahrten und Marienfrömmigkeit durch den Bau von Mariensäulen z. B. in Aachen und Trier zu manifestieren. Die Verwendung von religiösen Symbolen an der Oberfläche konnte die Geschichtsschreibung nicht davon abhalten, soziale und ökonomische Dimensionen (in der Industrialisierung marginalisierte Landbevölkerung findet im Klerus eine politische Stimme), politische Gründe (durch das allgemeine Wahlrecht erhielten katholische Parteien eine hohe Zahl von Stimmen) neben anderen Faktoren für das Verständnis miteinzubeziehen.

Mit den Muslimen und Europa verhält es sich strukturell vielleicht ähnlich. Mit ihren Lebensgewohnheiten, kulturellen und religiösen Traditionen unterscheiden sie sich von der Mehrheitsbevölkerung, deren religiöse Traditionen allmählich schwächer werden. Dass der Faktor Religion bei vielen Muslimen einen hohen Anteil an der Identität hat, kommt hinzu, wird aber verstärkt durch die Schaffung einer Opposition „Europa versus Islam“. Dem Text des Korans kommt auf der symbolischen Ebene eine recht große Bedeutung zu - größere Bedeutung sicherlich, als er innerhalb des theologischen Systems eines traditionellen Islams tatsächlich einnimmt. Skeptiker haben den Koran im Verdacht, mit dem Grundgesetz unvereinbar zu sein; muslimische Gruppen verkürzen ihre eigene Tradition auf einen einzigen Text. Jenseits aller symbolischen Konflikte, bei denen sich „Koran“ und „Europa“ gegenüberstehen, werden Rechtsstaatlichkeit, Gewaltenteilung, Demokratie, Religionsfreiheit und Bildungswesen in Europa von Muslimen hier und im Nahen Osten bewundert. Die im Entstehen begriffene Lebenswelt eines Islams in Deutschland wird ihre eigene Dynamik entfalten. Wie bei allen gesellschaftlichen und politischen Prozessen ist dies ein langer Weg, bei dem Konflikte und Debatten zur Natur der Sache gehören.

Aus der historischen Perspektive betrachtet steht Europa auf dem Gebiet der Philosophie, der Medizin sowie anderer griechischer Wissenschaften mit der arabischen Welt in enger Verbindung. Religiöse spätantike Texttraditionen jüdischer oder christlicher Prägung finden ein Echo in dem arabischen Text des Korans und stehen in einer hochinteressanten Verbindung. 
Die Vorstellung von einem jüdisch-christlichen Europa ist historisch gesehen sehr problematisch und wissenschaftlich letztlich nicht haltbar. Die Grundlagenforschung zum Text des Korans hat noch einen weiten Weg vor sich, voreilige Hypothesen spielen aus außerwissenschaftlichen Gründen in der breiten Debatte zwar gelegentlich eine Rolle, sind in ihrer Polemik aber am Ende schädlich für die gesellschaftliche Debatte. Europa mit seinen Wurzeln in der Antike und im Christentum muss sich historisch-kulturell im Rückblick in die komplexe Beziehungsgeschichte zur arabischen Welt und zum Islam neu positionieren.

\section{Literatur}

Bauer, Thomas, Die Kultur der Ambiguität: eine andere Geschichte des Islams, Berlin 2011.

Becker, Carl-Heinrich, Der Islam als Problem, Der Islam 1 (1910), 1-21.

Ben-Sasson, Menahem, Al-Andalus: Das „Goldene Zeitalter“ der spanischen Juden - kritisch besehen. In: Christoph Cluse (Hrsg.), Europas Juden im Mittelalter. Beiträge des internatinoalen Symposiums in Speyer vom 20. bis 25. Oktober, Trier 2004, S. 139-153.

Bergsträßer, Gotthelf, Plan eines Apparatus Criticus zum Koran, München 1930.

de Boer, Jan-Hendryk, Einfluss der arabischen Wissenschaften im europäischen Mittelalter; Sammelrezension von Al-Khalili, Jim: Im Haus der Weisheit. Die arabischen Wissenschaften als Fundament unserer Kultur, Frankfurt am Main 2011.

Brady, David, The Book of Revelation and the Qur'an: Is there a possible literary relationship?, Journal of Semitic Studies 23 (1978), S. 216-225.

Burgmer, Christoph (Hrsg.), Streit um den Koran, die Luxenberg-Debatte: Standpunkte und Hintergründe, Berlin ${ }^{3} 2007$.

Crone, Patricia und Cook, Michael A., Hagarism the making of the Islamic world, Cambridge 1977.

Déroche, François, La transmission écrite du Coran dans les débuts de l'Islam, Leiden 2009.

van Ess, Josef, Verbal Inspiration? Language and Revelation in Classical Islamic Theology, in: Stefan Wild (Hrsg.): The Quran as Text, Brill Leiden 1996, 177-194.

Flores, Alexander, Noch einmal: Thomas Bauer: Kultur der Ambiguität, inamo Informationsprojekt Naher und Mittlerer Osten Nr. 71, Berlin 2012.

Fraenkel, Siegmund, Die aramäischen Fremdwörter im Arabischen, Hildesheim u. a. 1962.

Franke, Ute und Joachim Gierlichs (Hrsg.), Roads of Arabia, The archaeological treasures of Saudi Arabia, Ausstellungskatalog, Tübingen/Berlin 2011.

Franke, Ute und Joachim Gierlichs (Hrsg.), Roads of Arabia, Archäologische Schätze aus Saudi-Arabien, Tübingen/Berlin 2011. 
Fück, Johann, Die Originalität des arabischen Propheten, in: Zeitschrift der Deutschen Morgenländischen Gesellschaft (ZDMG) 40 (1936), S. 515-516.

Goldziher, Ignaz, Zum islamischen Bilderverbot, in: Zeitschrift der Deutschen Morgenländischen Gesellschaft (ZDMG), Band 74, 1920.

Gougenheim, Sylvain, Aristote au Mont Saint-Michel. Les racines grecques de l'Europe chrétienne, Paris 2008.

Gougenheim, Sylvain, Aristoteles auf dem Mont Saint-Michel. Die griechischen Wurzeln des christlichen Abendlandes, deutsch von Jochen Grube, Darmstadt 2011.

Groß, Markus und Ohlig, Karl-Heinz (Hrsg.), Schlaglichter: Die beiden ersten islamischen Jahrhunderte, Berlin 2008 (INÂRAH, Schriften zur frühen Islamgeschichte und zum Koran, 3).

Groß, Markus und Ohlig, Karl-Heinz (Hrsg.), Vom Koran zum Islam, Berlin 2009 (INÂRAH, Schriften zur frühen Islamgeschichte und zum Koran, 4).

Groß, Markus und Ohlig, Karl-Heinz (Hrsg.), Die Entstehung einer Weltreligion I. Von der koranischen Bewegung zum Frühislam. Berlin u. Tübingen, 2 Bände. Band I: 2010. (INÂRAH, Schriften zur frühen Islamgeschichte und zum Koran, 5;) Band II: 2012. (INÂRAH, Schriften zur frühen Islamgeschichte und zum Koran, 6.)

Gutas, Dimitri, Greek Thought, Arabic Culture. The Graeco-Arabic Translation Movement in Baghdad and Early Abbasid Society (2nd-4th / 8th-10th centuries), London and New York 1998.

Haridi, Alexander, Das Paradigma der „islamischen Zivilisation“ - oder die Begründung der deutschen Islamwissenschaft durch Carl Heinrich Becker (1876-1933), Würzburg 2005.

Haverkamp, Alfred, Christliches und jüdisches Europa im Mittelalter, Kolloquium zu Ehren von Alfred Haverkamp, hrsg. v. Lukas Clemens und Sigrid Hirbodian, Red. Miriam Weiss, Trier 2011.

Horovitz, Josef, Koranische Untersuchungen. Studien zur Geschichte und Kultur des islamischen Orients, Heft 4, Berlin u. a. 1926.

Hoyland, Robert, Arabia and the Arabs from the Bronze Age to the Coming of Islam. London/New York 2001.

Hunke, Sigrid, Herkunft und Wirkung fremder Vorbilder auf den deutschen Menschen, Maschinenschriftliche Dissertation, Berlin 1941.

Hunke, Sigrid, Allahs Sonne über dem Abendland - Unser arabisches Erbe, Stuttgart 1960.

Ibn Warraq, Why I Am Not a Muslim, Amherst 1995.

Ibn Warraq (Hrsg.), What the Koran Really Says: Language, Text and Commentary, Amherst 2002.

Ibn Warraq (Hrsg.), Which Koran?: Variants, Manuscripts, Linguistics, Amherst 2011. 
Köster, Barbara, Der missverstandene Koran: Warum der Islam neu begründet werden muss, Berlin 2010.

Lüling, Günter Kritisch-exegetische Untersuchung des Qur'antextes, Erlangen 1970 (Inaugural-Dissertation).

Lüling, Günter, Über den Ur-Qur'ān, Erlangen 1974.

Lüling, Günter, Über den Ur-Koran, Ansätze zur Rekonstruktion der vorislamisch-christlichen Strophenlieder im Koran, Erlangen 1993.

Luxenberg, Christoph, Die syro-aramäische Lesart, ein Beitrag zur Entschlüsselung der Koransprache, Berlin 2000.

Luxenberg Christoph, Weihnachten im Koran, in: imprimatur, Trier 2003.

Luxenberg Christoph, Der Koran zum 'islamischen Kopftuch', in: imprimatur, Trier 2004.

Luxenberg Christoph, Neudeutung der arabischen Inschrift im Felsendom zu Jerusalem, in: Karl-Heinz Ohlig, Gerd R. Puin (Hrsg.), Die dunklen Anfänge - Neue Forschungen zur Entstehung und frühen Geschichte des Islam, Berlin 2005.

Luxenberg Christoph, Relikte syro-aramäischer Buchstaben in frühen Korankodizes im hejazi- und kufi-Duktus, in: Karl-Heinz Ohlig (Hrsg,), Der frühe Islam - eine historisch-kritische Rekonstruktion anhand zeitgenössischer Quellen, Berlin 2007.

Luxenberg, Christoph, Die syrische Liturgie und die "geheimnisvollen Buchstaben“ im Koran. Eine liturgievergleichende Studie. in: Markus Groß und Karl-Heinz Ohlig (Hrsg.), Schlaglichter, Die beiden ersten islamischen Jahrhunderte, Bd. 3, Berlin 2008.

Marx, Michael, Ein neuer Impuls für die Erforschung des Korans, inamo Informationsprojekt Naher und Mittlerer Osten Nr. 33, Berlin 2003, S. 45-47.

Marx, Michael, Ein neuer Impuls für die Erforschung des Korans Teil II, inamo Informationsprojekt Naher und Mittlerer Osten Nr. 34, Berlin 2003, S. 50-52.

Marx, Michael, Ein Koran-Forschungsprojekt in der Tradition der Wissenschaft des Judentumes: Zur Problematik ds Akademienvorhabens Corpus Coranicum, in: Dirk Hartwig u. a. (Hrsg.), Im vollen Licht der Geschichte. Die Wissenschaft des Judentums und die Anfänge der kritischen Koranforschung, Würzburg 2008, S. 41-54.

Marx, Michael, Bahira-Legende, Dante und Luxenberg - Von verschiedenen Koranwahrnehmungen, in: Christoph Burgmer (Hrsg.), Streit um den Koran, Berlin 2004, S. 112129.

Marx, Michael und Sinai, Nicolai, Islamische und Jüdische Hermeneutik als Kulturkritik. Historische Sondierungen und methodische Reflexionen zur Korangenese - Wege zur Rekonstruktion des vorkanonischen Korans, Berlin 2004 (Konferenzbericht).

Motzki, Harald, The Origins of Islamic Jurisprudence: Meccan Fiqh Before the Classical Schools, Leiden 2002.

Mulder, Eildert und Milo, Tom, De onstreden bronnen van het Islam, Amsterdam 2008.

Ohlig, Karl-Heinz, Gefahren und Chancen im Euro-Islam. Eine kritische Besinnung auf die Fundamente ist notwendig, in: Imprimatur Heft 4, Trier 2002. 
Ohlig, Karl-Heinz, Eine Revolution der Koran-Philologie, Zum Buch von Christoph Luxenberg, Die syro-aramäische Lesart des Koran. Ein Beitrag zur Entschlüsselung der Koransprache. Berlin (Das arabische Buch) 2000, 311 S.“, in: Imprimatur, Heft 5 Trier 2000.

Ohlig, Karl-Heinz, Zur Entstehung und Frühgeschichte des Islam, in: Aus Politik und Zeitgeschichte 26/27 vom 21.06.2007.

Ohlig, Karl-Heinz (Hrsg.), Der frühe Islam, Berlin 2007 (INÂRAH, Schriften zur frühen Islamgeschichte und zum Koran, 2).

Ohlig, Karl-Heinz und Puin, Gerd-Rüdiger (Hrsg.), Die dunklen Anfänge: neue Forschungen zur Entstehung und frühen Geschichte des Islam, Berlin ${ }^{2} 2005$.

Paret, Rudi Die Entstehung des islamischen Bilderverbots, in: Kunst des Orients. Bd. XI, 1/2 (1976-1977), Wiesbaden. S. 158-181.

Pressburg, Norman G., What the Modern Martyr Should Know: Seventy-two grapes and not single virgin, The new Picture of Islam, 2012.

Rassoul, Muhammad Ahmad, Die ungefähre Bedeutung des Al-Qur'an Al-Karim, Düsseldorf 2009.

Ratzinger, Karl, Jesus von Nazareth: Erster Teil. Von der Taufe im Jordan bis zur Verklärung, Freiburg 2007.

Robin, Christian Julien, A propos des "Filles de Dieu", Semitica 52-53 (2007), 139-148.

Robin Christian. Himyar et Israël. In: Comptes-rendus des séances de l'Académie des Inscriptions et Belles-Lettres, 148e année, N. 2, 2004, 831-908.

Rosenthal, Franz, The History of Heinrich Speyer's Die biblischen Erzählungen im Qoran, in: Dirk Hartwig u. a. (Hrsg.), Im vollen Licht der Geschichte. Die Wissenschaft des Judentums und die Anfänge der kritischen Koranforschung, Würzburg 2008, S. 113116.

Rosenthal, Franz, Das Fortleben der Antike im Islam, Zürich/Stuttgart 1965.

Speyer, Heinrich, Die biblischen Erzählungen im Qoran, Gräfenheinichen 1931.

Sarrazin, Thilo, Deutschland schafft sich ab. Wie wir unser Land aufs Spiel setzen, München 2010.

Schmitt, Arno, Rezenzion von Thomas Bauer, Die Kultur der Ambiguität, eine andere Geschichte des Islam, inamo Informationsprojekt Naher und Mittlerer Osten Nr. 70, Berlin 2012.

Sinai, Nicolai, The Qur'an as Process", in: Angelika Neuwirth, Nicolai Sinai, Michael Marx (Hrsg.), The Qur'an in Context: Historical and Literary Investigations into the Qur'anic Milieu, Leiden 2009.

Strohmaier, Gotthard, Denker im Reich der Kalifen, Berlin 1979.

Strohmaier, Gotthard, Von Demokrit bis Dante. Die Bewahrung antiken Erbes in der arabischen Kultur, Hildesheim/Zürich/New York 1996. 
Strohmaier, Gotthard, Zwischen Islamismus und Eurozentrismus. Mosaiksteine zu einem Bild arabisch-islamischen Erbes, Wiesbaden 2012, Kap. 1 „Europa im schrägen Blick eines Gräko-Arabisten“, S. 1-11.

Strohmaier, Gotthard, Hellas im Islam. Interdisziplinäre Studien zur Ikonographie, Wissenschaft und Religionsgeschichte, Wiesbaden 2003.

al-Talhi, Dhaifallah und Mohammad al-Daire, Roman Presence in the Desert: A New Inscription from Hegra, Chiron 35, 2005.

Thor, Brad, The last patriot. A thriller, New York 2008.

Tisdall, William St. Clair, The Origins of The Koran: Classic Essays on Islam's Holy Book, 1911.

Updike, John, Terrorist: A novel, New York 2006.

Wansbrough, John, Quranic studies sources and methods of scriptural interpretation, Oxford 1977.

Wansbrough, John, The Sectarian Milieu: Content and Composition Of Islamic Salvation History, Oxford, 1978.

Weidner, Stefan, Aufbruch in die Vernunft. Islamdebatten und Islamische Welt zwischen 9/11 und den Arabischen Revolutionen, Bonn 2011, Kap. „Islam und Westen Hard oder Software?", S. 45-60.

Wick, Lukas, Islam und Verfassungsstaat. Theologische Versöhnung mit der politischen Moderne? Würzburg 2009.

\section{Zeitungsquellen}

Artikel: Ausländerbeauftragter findet Islam-Buch wichtig, Ostthüringer Zeitung, 09.09.2010.

Artikel: Thüringen schickt umstrittenen Ausländerbeauftragten in Ruhestand, Leipziger Volkszeitung vom 09.09.2010.

Higgins, Andrew, The lost archive: Missing for a half century, a cache of photos spurs sensitive research on Islam's holy text", Wall Street Journal, 12.01.2008.

Ibn Warraq, Virgins? What virgins?, The Guardian vom 12.01.2002.

Lau, Jörg, Keine Huris im Paradies, Die Zeit Nr. 21 vom 15.05.2003.

Roger-Pol Droit, Et si l'Europe ne devait pas ses savoirs à l'islam ? Le Monde 4.8.2008.

Ricklin, Thomas, Skandalbuch von Sylvain Gouguenheim Der Mittelalter-Sarrazin, Süddeutsche Zeitung vom 24.08.2011.

Schirrmacher, Frank Ein Buch fehlt, Frankfurter Allgemeine Zeitung vom 10.10.2007, Nr. 235, S. 1.

Schwanitz, Wolfgang G., Großäugige Trauben - Wenn aus Knaben Früchte werden, Der Tagesspiegel vom 19.02.2007.

Theil, Stefan, Challenging the Qur'an. A German scholar contends that the Islamic text has been mistranscribed and promises raisins, not virgins, Newsweek 27.7.2003. 


\section{Internetquellen (15.10.2012):}

Schreiner, Stefan, Das „christliche Europa“. Eine Fiktion, Qantara.de, http://de.qantara.de/ Eine-Fiktion/19611c20859i0p231/index.html, 8.8.2012.

\section{Rezensionen zu Luxenberg (2000)}

Baasten, Martin F. J., in: Aramaic Studies, Nr. 2.2, Leiden 2004, S. 268-272.

de Blois, François, in: Journal of Qur'anic Studies, Band V, Ausg. I, London 2003, S. 92-97. Hopkins, Simon, in: Jerusalem studies in Arabic and Islam, Nr. 28, Jerusalem 2003, S. 377380.

Horsten, Piet, in: islamochristiana 28, 2002. S. $310 \mathrm{f}$.

Jansen, Johannes J. G., in: Bibliotheca Orientalis, LX N³-4, mei-augustus 2003, Sp. 477480.

Maas, Wilhelm Maria, Der Koran - ein christliches Lektionar?, in: Novalis, Zeitschrift für spirituelle Entwicklung, Quern 2003, S. 18-22.

Neuwirth, Angelika, in: Journal of Qur'anic Studies, Band V (2003), Ausgabe I, Berlin 2003, S. 1-18.

Phenix, Robert R. Jr., Cornelia B. Horn, in: Hugoye. Journal of Syriac Studies 6-2003, 1. 